\title{
The Pore-Lipid Interface: Role of Amino-Acid Determinants of Lipophilic Access by Ivabradine to the hERG1 Pore Domain $\mathrm{s}$
}

\author{
Laura Perissinotti, ${ }^{1}$ Jiqing Guo, ${ }^{1}$ Meruyert Kudaibergenova, ${ }^{1}$ James Lees-Miller, \\ Marina Ol'khovich, Angelica Sharapova, German L. Perlovich, Daniel A. Muruve, \\ Brenda Gerull, Sergei Yu Noskov, and Henry J. Duff
}

Centre for Molecular Simulation, Department of Biological Sciences, University of Calgary, Calgary, Alberta, Canada (L.P., M.K., S.Y.N.); Libin Cardiovascular Institute of Alberta (J.G., J.-L.M., H.J.D.) and Snyder Institute for Chronic Diseases (D.A.M.), Cumming School of Medicine, University of Calgary, Calgary, Alberta, Canada; Institute of Solution Chemistry, Russian Academy of Sciences, Ivanovo, Russian Federation (M.O., A.S., G.L.P.); Department of Cardiac Sciences and Libin Cardiovascular Institute of Alberta, University of Calgary, Calgary, Alberta, Canada (B.G.); and Comprehensive Heart Failure Center and Department of Internal Medicine I, University Hospital Würzburg, Würzburg, Germany (B.G.)

Received December 21, 2018; accepted May 28, 2019

\section{ABSTRACT}

Abnormal cardiac electrical activity is a common side effect caused by unintended block of the promiscuous drug target human ether-à-go-go-related gene (hERG1), the pore-forming domain of the delayed rectifier $\mathrm{K}^{+}$channel in the heart. hERG1 block leads to a prolongation of the QT interval, a phase of the cardiac cycle that underlies myocyte repolarization detectable on the electrocardiogram. Even newly released drugs such as heart-rate lowering agent ivabradine block the rapid delayed rectifier current $\mathrm{I}_{\mathrm{Kr}}$, prolong action potential duration, and induce potentially lethal arrhythmia known as torsades de pointes. In this study, we describe a critical drug-binding pocket located at the lateral pore surface facing the cellular membrane. Mutations of the conserved M651 residue alter ivabradine-induced block but not by the common hERG1 blocker dofetilide. As revealed by molecular dynamics simulations, binding of ivabradine to a lipophilic pore access site is coupled to a state-dependent reorientation of aromatic residues $\mathrm{F} 557$ and $\mathrm{F} 656$ in the S5 and S6 helices. We show that the M651 mutation impedes state-dependent dynamics of F557 and F656 aromatic cassettes at the protein-lipid interface, which has a potential to disrupt drug-induced block of the channel. This fundamentally new mechanism coupling the channel dynamics and small-molecule access from the membrane into the hERG1 intracavitary site provides a simple rationale for the well established state-dependence of drug blockade.

\section{SIGNIFICANCE STATEMENT}

The drug interference with the function of the cardiac hERG channels represents one of the major sources of drug-induced heart disturbances. We found a novel and a critical drug-binding pocket adjacent to a lipid-facing surface of the hERG1 channel, which furthers our molecular understanding of drug-induced QT syndrome.

\section{Introduction}

The cardiac action potential is primarily generated by sodium and calcium channels, which depolarize the membrane potential, and by potassium channels, which repolarize

This work was supported by the Canadian Institutes of Health Research Project Grant FRN-CIHR: 156236 (to J.G., J.L.-M., and H.J.D.); the National Institutes of Health [Grant R01HL128537-01 (S.Y.N., J.G., L.P., and M.K.)]; and the Discovery grant from the Natural Scientific and Engineering Research Council of Canada (to H.J.D.). Computational support for this work was partially provided by West-Grid Canada through a resource allocation award to S.Y.N. M.K. was supported by a Queen Elizabeth II graduate scholarship. Anton 2 computer time was provided by the Pittsburgh Supercomputing Center (PSC) through Grant R01GM116961 from the National Institutes of Health. The Anton 2 machine at PSC was generously made available by D.E. Shaw Research.

${ }^{1}$ L.P., J.G., and M.K. contributed equally to this work.

https://doi.org/10.1124/mol.118.115642.

S This article has supplemental material available at molpharm.aspetjournals.org. the membrane potential and terminate the action potential (Nerbonne and Kass, 2005). Gene mutations, age-related factors, and drug-induced toxicity are all linked to various perturbation of action potentials, leading to potential lethal disorders of heart rhythm (arrhythmias) (Chiamvimonvat et al., 2017). Several $\mathrm{K}^{+}$-selective channels were identified as major determinants of proarrhythmic activity and main targets in antiarrhythmic drug development. Perhaps the most impactful of these is the human ether-a-go-go-related gene 1 (hERG1 or Kv11.1) channel, the $\mathrm{K}^{+}$-selective channel carrying the rapid delayed rectifier current $\left(\mathrm{I}_{\mathrm{Kr}}\right)$ in myocytes (Trudeau et al., 1995). The physiologic role of $\mathrm{I}_{\mathrm{Kr}}$ is to repolarize the late phase of cardiac action potential; hence, currents carried by hERG1 that contain human mutations are linked to arrhythmias (Gustina and Trudeau, 2009; Vandenberg et al., 2012). Likewise, pharmacological blockage of the $\mathrm{I}_{\mathrm{Kr}}$ can lead to

ABBREVIATIONS: cryo-EM, cryoelectron microscopy; hEAG1, human ether à go-go 1 gene in reference to $\alpha$-subunit of Kv10.1 $\mathrm{K}^{+}$channel; hERG1, the human ether-à-go-go-related gene in reference to $\alpha$-subunit of Kv11.1 $\mathrm{K}^{+}$channel; IFD, Induced-Fit Docking; I herg1, current carried by hERG1; $I_{K r}$, rapid delayed rectifier current; MD, molecular dynamics; WT, wild-type. 
a prolongation of the QT interval (phase 3 repolarization), causing a drug-induced long-QT syndrome (Compton et al., 1996; Roden et al., 1996; Splawski et al., 1997; Huang et al., 2001; Vandenberg et al., 2001). Drug-induced block of hERG1 and its associated prolongation of the QT interval and proarrhythmia has resulted in hERG1 being one of the most studied ion channels (Numaguchi et al., 2000; Witchel, 2011; Sanguinetti, 2014). Recent high-throughput screening studies have provided additional evidence for the central role of hERG1 blockade in drug-safety assessments (Di Veroli et al., 2013a).

Despite the plethora of experimental data available for drug interactions with the hERG1 channel, a-priori prediction of the cardiotoxic potential of a novel compound during a preclinical developmental stage is a complex and a challenging task. The torsadogenicity is an emergent and a complicated property that depends on several factors: the conformational state of the channel being targeted by the compound (Chen et al., 2002; Stork et al., 2007; Lees-Miller et al., 2015; Wu et al., 2015), general kinetics of the drug access mechanisms (Guo et al., 2005; Di Veroli et al., 2013b; Hill et al., 2014), the solubility of the drug in the membrane (Wacker and Noskov, 2018), patient-to-patient variance in isoform composition of hERG1 (hERG1a/hERG1b) (Sale et al., 2008), and isoformspecific interactions with blockers (Abi-Gerges et al., 2011). Given these factors, understanding molecular determinants of drug-induced QT-prolongation remains one of the fundamental and elusive problems in the field of molecular pharmacology. Even newly released "cardio-safe" drugs entering the market, such as ivabradine, are found to block the $\mathrm{I}_{\mathrm{Kr}}$, prolong action potential duration, and can contribute to a potentially lethal drug-induced arrhythmia known as torsades de pointes (Duff et al., 1995; Lees-Miller et al., 2000, 2015; Chen et al., 2002; Melgari et al., 2015).

Ivabradine is commonly prescribed as a blocker of $\mathrm{I}_{\mathrm{f}}$ (funny current) and acts as a heart-rate lowering agent for symptomatic management of the chronic heart failure. However, over a similar range of concentrations, the drug also blocks hERG; thus, ivabradine prolongs phase 3 of the action potential and has been reported to induce torsades de pointes when applied in a poly-pharmaceutical context (Hancox et al., 2015; Melgari et al., 2015; Frommeyer et al., 2017). In a previous study, by combining electrophysiology and molecular modeling techniques, we emphasized the importance of the lipophilic interface and high-affinity state-dependent blockade of hERG1 by ivabradine (Lees-Miller et al., 2015). The molecular simulations performed on the homology model of hERG1 pore domain in open and closed states indicated that ivabradine may bind to a lipid-facing binding pocket centered at the M651 residue (Lees-Miller et al., 2015). However at the time, findings were limited owing to the lack of structural information on the organization of the pore domain of the hERG1 channel. In 2017, a high-resolution cryoelectron microscopy (cryo-EM) structure (3.4 $\AA$ ) of a highly homologous neuronal human ether à go-go 1 gene (hEAG1) channel was resolved with a voltage-sensor in a depolarized (open) state, but with the pore domain closed. Later in the same year, the high-resolution structure of the hERG1 channel $(3.2 \AA)$ was reported in the open state (Whicher and MacKinnon, 2016; Wang and MacKinnon, 2017) (Fig. 1). These new structural atomic details provided the opportunity to unravel the potential lipophilic access mechanisms for the ivabradine-induced block of hERG1, to assess the impact of mutations at the M651 site in the distal S6, and to understand the dynamics of allosteric coupling between residues involved in the high-affinity channel block.

Herein, we report an in-depth study that provides direct evidence of ivabradine's interaction in a state-dependent manner with lipid-facing residues as part of the hERG1 blockade process. We assessed whether mutations of the lipid-facing residues, when coupled with C-type inactivation impacting mutations, affect the concentration of ivabradine required to block the hERG1 current. To reveal mechanisms involved in coupling dynamics of the lipid-facing residues with respect to the aromatic cassette involved in high-affinity drug block by various compounds (Fig. 1), we performed several multi-microsecond molecular dynamics (MD) simulations of the wild-type (WT) and mutated forms of hERG1 in conjunction with molecular biology and electrophysiological studies. The MD simulations in combination with the ensemble docking simulations allowed us to map molecular details of the probable lipophilic access pathway of ivabradine and potentially explain the drug's dependence on the inactivation process of hERG1 channel. Our results show that ivabradine's binding at the vicinity of the lipid-facing residue M651 is directly coupled to the conformational dynamics (reorientation) of the aromatic cassettes (F656 and F557) in the S6 (Fig. 1, A and B). Disruption of this allosteric coupling between drug binding on the lipid-facing surface and conformational dynamics of F656/F557 was shown to greatly attenuate ivabradine blockade.

\section{Materials and Methods}

Molecular Biology. Methods for site-directed mutagenesis have been previously reported (Lees-Miller et al., 2015; Wang et al., 2016). The hERG1 constructs were transfected into mammalian human embryonic kidney cells. Single- and double-mutant constructs of hERG1 were produced using conventional overlap polymerase chain reaction with primers synthesized by Sigma Genosys (Oakville, ON, Canada) and sequenced using Eurofins MWG Operon (Huntsville, $\mathrm{AL})$. Constructs were linearized with $\mathrm{XbaI}$ restriction endonuclease, and cRNA was transcribed in vitro using the mMessage mMachine T7 Ultra cRNA transcription kit (Ambion, Austin, TX).

General Setup for Electrophysiological Recordings. The extracellular solution contained (in millimolars) $\mathrm{NaCl} 140, \mathrm{KCl} 5.4$, $\mathrm{CaCl}_{2} 1, \mathrm{MgCl}_{2} 1, \mathrm{HEPES} 5$, and glucose 5.5 ; the $\mathrm{pH}$ of the solution was adjusted and kept at 7.4 with $\mathrm{NaOH}$. Micropipettes were pulled from borosilicate glass capillary tubes on a programmable horizontal puller (Sutter Instruments, Novato, CA). The pipette solution contained the following: $10 \mathrm{mM} \mathrm{KCl}, 110 \mathrm{mM} \mathrm{K}$-aspartate, $5 \mathrm{mM} \mathrm{MgCl}_{2}, 5 \mathrm{mM}$ $\mathrm{Na}_{2} \mathrm{ATP}, 10 \mathrm{mM}$ EGTA (ethylene glycol-bis(-aminoethyl ether)$N, N, N, N$ tetraacetic acid), $5 \mathrm{mM}$ HEPES, and $1 \mathrm{mM} \mathrm{CaCl} 2$; the solution was adjusted to $\mathrm{pH} 7.2$ with $\mathrm{KOH}$. Standard patch-clamp methods were used to measure the whole-cell currents of hERG1 mutants expressed in human embryonic kidney 293 cells using the AXOPATCH 200B amplifier (Axon Instruments). Unless otherwise indicated, the tail currents were recorded when the voltage was returned to $-100 \mathrm{mV}$ from $+50 \mathrm{mV}$. Transfected human embryonic kidney cells were patched to record the hERG1 currents. Ivabradine was directly dissolved into the Tyrode solution right before the experiments. The solutions were used for the next 2 hours during the experiments. The stock solution of $100 \mu \mathrm{M}$ ivabradine was prepared in the extracellular solution. Fresh stock solutions of ivabradine were prepared weekly.

Voltage-Dependence of Activation. From a holding potential of $-80 \mathrm{mV}$ cells were depolarized for 1 second to a range of voltages from -100 to $+40 \mathrm{mV}$ followed by a step to $-100 \mathrm{mV}$ ( 1 second) to 
A
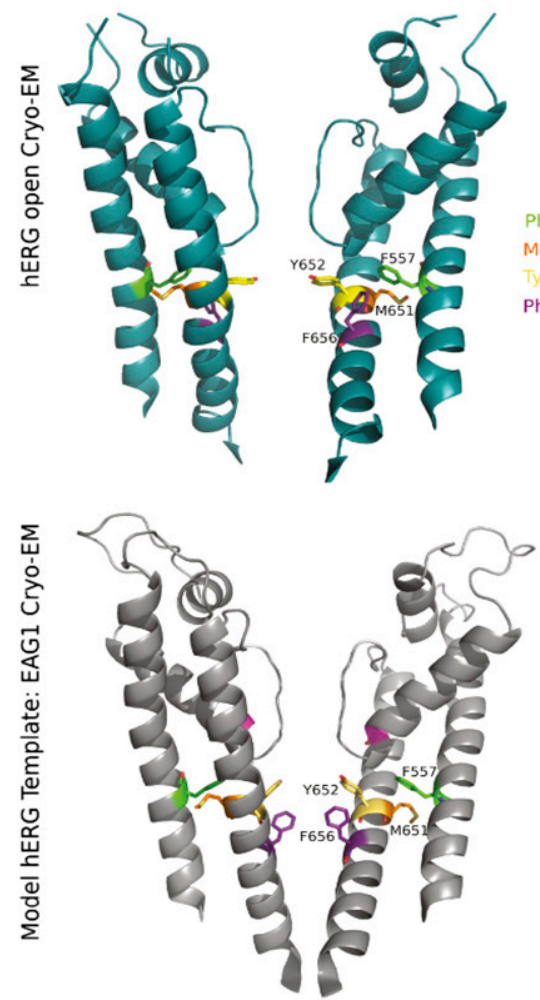

B
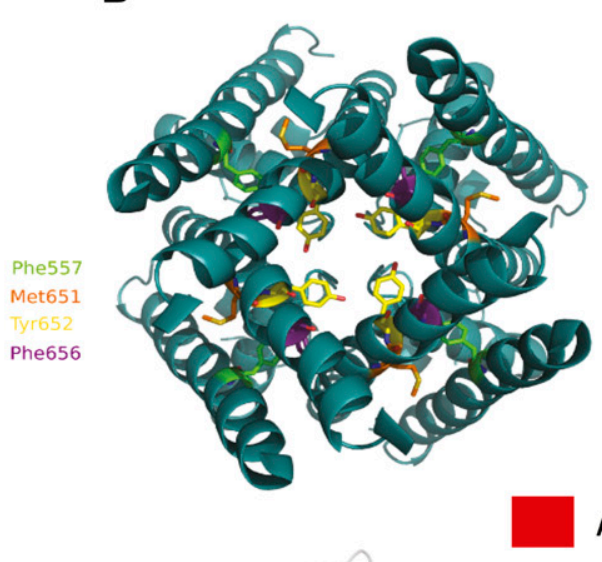

C

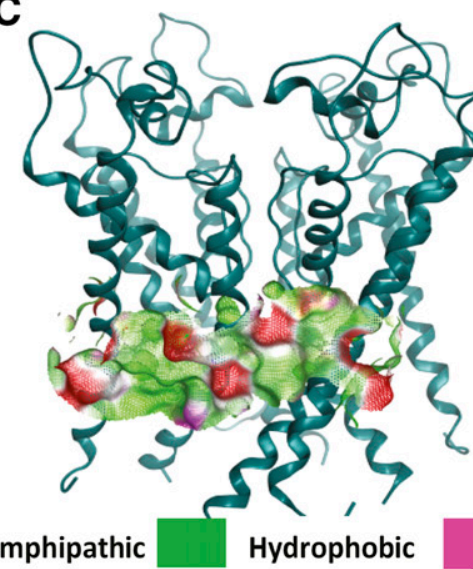

Polar

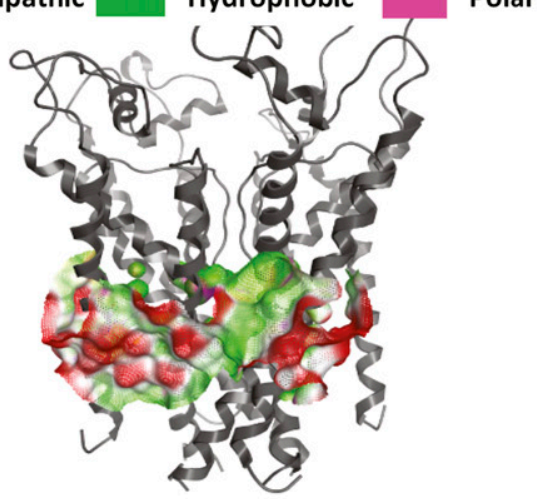

Fig. 1. Structural organization of drug binding site in the intracellular cavity of hERG1 channel. (A) Organization of the hERG1 pore domain (two subunits are shown for clarity) in the open (top) and closed (bottom) states, respectively. The location of key residues involved in the state-dependent dynamics of membrane-facing side windows is shown in color-coded stick mode. (B) Access pathway to the intracellular cavity of hERG1 channel from the intracellular milieu. The differences in orientation of key hydrophobic residues involved to ivabradine-induced block are shown for the open (top) and closed (bottom) states, respectively. (C) The trajectory-averaged iso-surfaces available for the drug's lipophilic access to the intracellular cavity. The residue-based color coding was used to highlight protein-membrane interfaces present in the open (top) and closed (bottom) states of the channel, respectively.

record the tail currents. The isochronal tail-current-voltage plots were fit to a single Boltzmann function (1):

$$
\frac{I}{I_{\max }}=\frac{1}{\left(1+\exp \left[\left(V_{1 / 2}-V_{m}\right) / k\right]\right)}
$$

where $I / I_{\max }$ is the normalized current, $V_{1 / 2}$ is voltage of the halfmaximal activation, $k$ is the slope factor, and $V_{m}$ is the membrane potential.

Analysis of Deactivation. Deactivation of hERG1 tail currents was measured by activating channels at $+40 \mathrm{mV}$, followed with a short (5-millisecond) repolarization step to $-120 \mathrm{mV}$ and deactivating steps at $-120,-100,-60$, and $-40 \mathrm{mV}$. Currents at different voltages were normalized and fitted. The fitted data were averaged $(n=10)$.

Statistical Analysis of Electrophysiological Experiments. Statsview (Abacus Concepts, Berkeley, CA) or QTI plot (Vasilef, 2013), Grace (http://plasma-gate.weizmann.ac.il/Grace/) were used to analyze the data. The null hypothesis of this study predicted no difference between the $\mathrm{IC}_{50}$ values of the single mutation to the double mutations assessed. The null hypothesis was rejected when the $P$ value was $<0.05$ as evaluated by a one-way analysis of variance with Tukey test. The exact $P$ values for data in Fig. 4 are presented in the Supplemental Table 1. All variance measures (bars) for electrophysiological data are shown as S.D. The study was exploratory, thus there was no a priori reason to consider whether there was an additive or subtractive interaction. In addition, we acknowledge that there may be other mutations, unknown to us at this time, which could be relevant. All of the comparisons were prespecified and all of the comparisons are reported. A priori, we generally required a minimum of $n=2$ independent experiments for each point on the $\mathrm{IC}_{50}$ curve. However, the $n$ values near the $\mathrm{IC}_{50}$ point and near the maximum blockade have a major impact on the reliability of the $\mathrm{IC}_{50}$ estimate. Frequently we increased the $n$ values at these putative points to be more certain about the reliability of the measurement. The $n$ values for each point of the concentration-response relationship are presented in the figure legends. J.G. executed the experiments and analyzed the experimental data, hence he was not blinded. However, each experiment was reviewed by a small committee of individuals to assess quality of the records at weekly laboratory meetings (J.G. and H.J.D.) and on a monthly basis (H.J.D. and S.Y.N.) to review raw experimental data.

Dofetilide and Ivabradine Water/Hexane Partitioning Experiments. The distribution coefficient for dofetilide and ivabradine were determined by the use of a classic shake-flask method detailed in our previous publications (Perlovichl and Bauer-Brandl, 2003; Perlovich et al., 2006; Blokhina et al., 2016). Prior to conducting the experiments, both solvents were mutually saturated to reach equilibrium by slow stirring into a biphasic system for 2 days. Ivabradine and dofetilide were dissolved in a buffer at $\mathrm{pH}=7.4$ and then were added to a hexane solution. To ensure complete equilibration of the system as indicated by the absence of turbidity on each phase, the flasks were shaken for about 48 hours in a thermostatic water bath at 293.15, $298.15,303.15,310.15$, and $313.15 \mathrm{~K}$. After reaching equilibration in the system, the samples from the lower phase were carefully removed with syringes for analysis. The molar concentrations of the dofetilide and ivabradine in the buffer phase were measured by a Cary-50 spectrophotometer (Varian) with an accuracy of $2 \%-4 \%$. The experimental results are reported as an average value of at least three 
replicated experiments. The accuracy of the distribution coefficients were verified by comparing the starting mass of a compound and the total mass of the compound distributed in the two phases. The reproducibility of the measured concentrations was under $0.1 \%$, and the maximum deviations from the average value were $<0.15 \%$. The ratio of the compound equilibrium concentration in mole fraction in the hexane phase $\left(x_{H}\right)$ to those in the aqueous phase $\left(x_{B}\right)$ was determined using the hexane/buffer distribution coefficient in the following form:

$$
D_{H / B}=x_{H} / x_{B}
$$

$D_{H / B}$ is the phase equilibrium constant for a drug distributed in the hexane phase and the saturated buffer phase.

The standard Gibbs energy of transfer $\Delta_{\mathrm{tr}} G^{o}$ from the buffer into an organic solvent was calculated by using:

$$
\Delta_{\mathrm{tr}} G^{o}=-R T D_{H / B}
$$

The temperature dependence of distribution (van't Hoff method) was employed to obtain the enthalpy of transfer $\Delta_{\mathrm{tr}} H^{o}$ :

$$
\frac{\mathrm{d}\left(\ln D^{*}\right)}{\mathrm{d} T}=\frac{\Delta_{\mathrm{tr}} H^{o}}{R T^{2}}
$$

The entropy of transfer $\Delta_{\mathrm{tr}} S^{o}$ was calculated from:

$$
\Delta_{\mathrm{tr}} S^{o}=\left(\Delta_{\mathrm{tr}} H^{o}-\Delta_{\mathrm{tr}} G^{o}\right) / T
$$

In-depth details on the partitioning experiments and quality controls are provided in the Supplemental Materials.

Molecular Dynamics Simulations. The three-dimensional structure for the open state of the channel in this study is the recently published high-resolution cryo-EM structure of the transmembrane domain of hERG1channel (PDB ID 5VA2). The structure was truncated before the Per-Arnt-Sim domain (PAS) and after cyclic nucleotidebinding domain domains as described in our previous publication (Wacker et al., 2017; Perissinotti et al., 2018). The basis for the threedimensional structure for the closed-state hERG1 channel is the homology modeling of hEAG1 with template derived from the cryo-EM structure (PDB ID 5K7L) solved at $3.78 \AA$ resolution. The SWISSMODEL program (Kopp and Schwede, 2004) was used to develop the hERG1 closed homology model from the available hEAG1 channel structure. Sequence alignment was performed using the CLUSTALW algorithm (Thompson et al., 1994). The sequence similarity between hERG1 and hEAG1 channels for the pore domain (S5-S6) is over 75\% (Wacker et al., 2017). The detailed analysis of two structures was published recently (Vandenberg et al., 2017; Wacker et al., 2017). The main differences between pore domains of the two channels are located in the extended turret region connecting S5 to the pore helix. The following three-step protocol was adapted to model missing residues and flexible elements: 1 ) threading for generation of initial models on the basis of template structure by copying coordinates over the aligned regions (for closed states), 2) low-resolution ROSETTA loop modeling using the cyclic coordinate descent method, 3) highresolution all-atom refinement and selection of models on the basis of ROSETTA clustering (Bender et al., 2016).

Models of the protein were generated from the alignment in a stepwise manner. CHARMM-GUI (Jo et al., 2008) was used to prepare protein-dipalmitoylphosphatidyl choline lipid bilayer complexes solvated in $150 \mathrm{mM} \mathrm{KCl}$ aqueous solution using CHARMM-36 forcefield and TIP3P water model (Jorgensen et al., 1983; MacKerell et al., 1998; Noskov et al., 2004; Noskov and Roux, 2008; Klauda et al., 2010; Best et al., 2012). The fully assembled systems were equilibrated for 10 nanoseconds using NAMD2.10 (Phillips et al., 2005) and then subjected to production runs with the Anton 2 supercomputer. The production runs were performed for 1.0-2.5 microseconds, each with CHARMM36M (Huang et al., 2017) force-field to assess structural dynamics of residues involved in lipophilic access in hERG1 WT and selected mutants. The production runs were executing in a semiisotropic (NPaT) ensemble at a temperature of $315 \mathrm{~K}$ maintained by the Nosé-Hoover thermostat (Martyna et al., 1994). The time-step for production runs was set to 2 femtoseconds, and trajectories were saved every 240 picoseconds. Nonbonded and long-range electrostatic interactions were evaluated every 2 and 6 femtoseconds, respectively. Long-range electrostatics was calculated using the k-Gaussian-Ewald method implemented to enhance performance on Anton 2 platform (Shan et al., 2005; Shaw et al., 2014) with a $64 \AA \times 64 \AA \times 64 \AA$ grid. SHAKE was used to constrain all bonds involving hydrogen atoms. All of the subsequent trajectory analysis was performed using the CHARMM c40b2 program package (Brooks et al., 2009).

Molecular Docking Protocols. Ivabradine in neutral and charged states was docked in-silico to the hERG1 open- and closedpore structures to establish binding modes. This was performed via the Induced-Fit Docking (IFD) approach available in the Schrödinger Suite (Schrodinger LLC, 2016). Schrödinger's IFD protocol uses Glide and Prime to exhaustively consider possible binding modes and the associated conformational changes within the receptor's active sites (Schrodinger LLC, 2016). In addition to the well established highaffinity binding pocket in the intracellular cavity of hERG1 channels, previous blinded docking studies revealed several alternative binding regions present in the hERG1 homology models and the cryo-EMderived structures (Lees-Miller et al., 2015; Saxena et al., 2016; Wacker et al., 2017). To map these alternative binding pockets as accurately as possible, we adapted the following two-step protocol. First, a blind docking was performed covering an entire receptor (e.g., pore and voltage-sensing domains regions of hERG1), and then elucidated common binding sites were selected for further studies with high-precision grid mapping. The SiteMap module of the Schrödinger molecular modeling package was used to generate finegrids for the subsequent precision docking. Following previously tested protocols, the grid was defined to 15 "site-points" for each pocket found during the blinded docking run (Schrodinger LLC, 2016). Then, site maps are cropped $10 \AA$ from the nearest site-point (Halgren, 2009). The scoring was carried out using Schrödinger's discretized version of the ChemScore empirical scoring function, resulting in a small number of best-refined poses, following which the best-docked protein-ligand complex was determined on the basis of a model energy score $\left(E_{\text {model }}\right)$ that combined the energy of the grid-score, the binding affinity predicted by GlideScore, and (for flexible docking) the internal strain energy for the model potential used to direct the conformational-search algorithm (Schrodinger LLC, 2016). The basis for the partial charges for the neutral form of the drug was the nonbonded parameters from the Optimized Potentials for Liquid Simulation Force Field (OPLS3) with parameters specifically optimized for drug-like molecules (Harder et al., 2016). For all docking simulations the funnel width was increased by adjusting the energy window to 5.0 , the CvdW cutoff was set to $10.0 \mathrm{kcal} / \mathrm{mol}$, and the clustering criteria was set to 0.75 and extra-precision (XP) (Friesner et al., 2006). In addition to the single-structure docking procedure, MD-generated ensembles for the open hERG1 transmembrane and mutant forms were used in an ensemble docking with ivabradine to account for the conformational dynamics of the pore domain. A similar protocol was used in a comparative study of a cationic ivabradine binding to the main intracavitary site present in the open state of hERG1.

Ensemble Docking Procedure. From the last 1.0 microseconds of the production MD trajectories, we randomly selected 25 frames spaced every 40 nanoseconds. The basis of the alignment of each frame was the position of the backbone atoms from the pore domain (residues 545-572 and 635-669). Glide was used with the XP ensemble docking (Friesner et al., 2006) with Schrodinger Small-Molecule Drug Discovery Suite 2018-2 (2016) as described above for the single-structure docking protocol. The ligand binding site defined in a single-structure receptor docking was the basis for the generation of each receptor grid. Each generated grid is made of two boxes: the inner box for searching docking space that defines acceptable volume for the ligand center to 
explore and the outer box for searching the space of valid poses that must contain all ligand atoms for which grid potentials are computed. The inner cubic box was fixed at the dimension of $15 \AA \times 15 \AA \times 15 \AA$ and the outer box was set to $30 \AA \times 30 \AA \times 30 \AA$. The number of selected docking poses per docking simulation was set to approx. 50 , and only poses with binding affinities of $<-3 \mathrm{kcal} / \mathrm{mol}$ were clustered. It is important to mention that grids generated by the SiteMap protocol were overlapping. Therefore, poses obtained from separate docking simulations were clustered into three sites on the basis of where the ligand was bound.

The first site corresponds to all poses found inside the intracellular cavity of the receptor (within approx. $3 \AA$ of the $\mathrm{C}_{\alpha}$ atoms of $\mathrm{Y} 652$ or F656); poses on the lipid-facing transmembrane domain (lipophilic, within approx. $3 \AA$ of the $\mathrm{C}_{\alpha}$ atom of M651) maps lipophilic site 2 and poses found between transmembrane segments of the pore domain (S5-S6) and the voltage sensing domain (S1-S4) map a putative "access path" site 3 .

\section{Results}

Rationale for the Selection of Mutants. Our previous in-silico screening of ivabradine binding to the hERG1 model representing open and closed states of the channel identified several potential binding modes (Lees-Miller et al., 2015). The best-scored binding poses for neutral and cationic ivabradine were clustered around a well known intracavitary site in the open state of hERG1; however, almost no binding in the internal cavity (Y652-F656) was observed for the closed-state model. Two aromatic residues in the S6 helix lining the intracavitary site that are most commonly associated with high-affinity blockade of hERG1 are Y652 and F656. Both residues are established as a critical determinant of hERG1induced block associated with proarrhythmia (Ficker et al., 1998; Lees-Miller et al., 2000; Mitcheson et al., 2000; Perry et al., 2010). Recent work of Saxena et al. (2016) emphasized an important role for another aromatic residue (F557) from the S5 helix. It was shown that F557 may be involved in the drug stabilization of hERG1 binding pocket along with the residues in the $\mathrm{S} 6$ helix. Therefore, we chose to include F557X along with classic aromatic cassette Y652X and F656X to screen for ivabradine binding to the well established intracellular site.

As for the M651 mutation, previous blinded docking studies showed the presence of a potential binding domain for ivabradine in close proximity to this residue, only in the open state of the channel (Lees-Miller et al., 2015). However, no electrophysiological experimental data on M651X mutants was reported in the previous report. The MD simulations also showed a favorable energetic partitioning of the neutral state ivabradine into the lipid bilayers. The comparison of in-silico models used by Lees-Miller et al. (2015) to the recently solved cryo-EM structures showed that the key structural elements in the pore domain (S5-S6) were accurately captured by ROSETTA-generated models of hERG1 (RMSD < $3.5 \AA$ A) (Wang et al., 2016) providing additional support for docking studies on hERG1 models. In this study we created the M651T mutation to examine its effects on ivabradine binding (Fig. 2). We reasoned that the substitution of methionine by the small polar amino acid (threonine) at the M651 site could disrupt the lipophilic binding or access route of ivabradine. We discovered that M651T expressed well in cells but the mutation slowed deactivation kinetics of the channel and suppressed the block induced by ivabradine (Fig. 2). This raised a possibility that
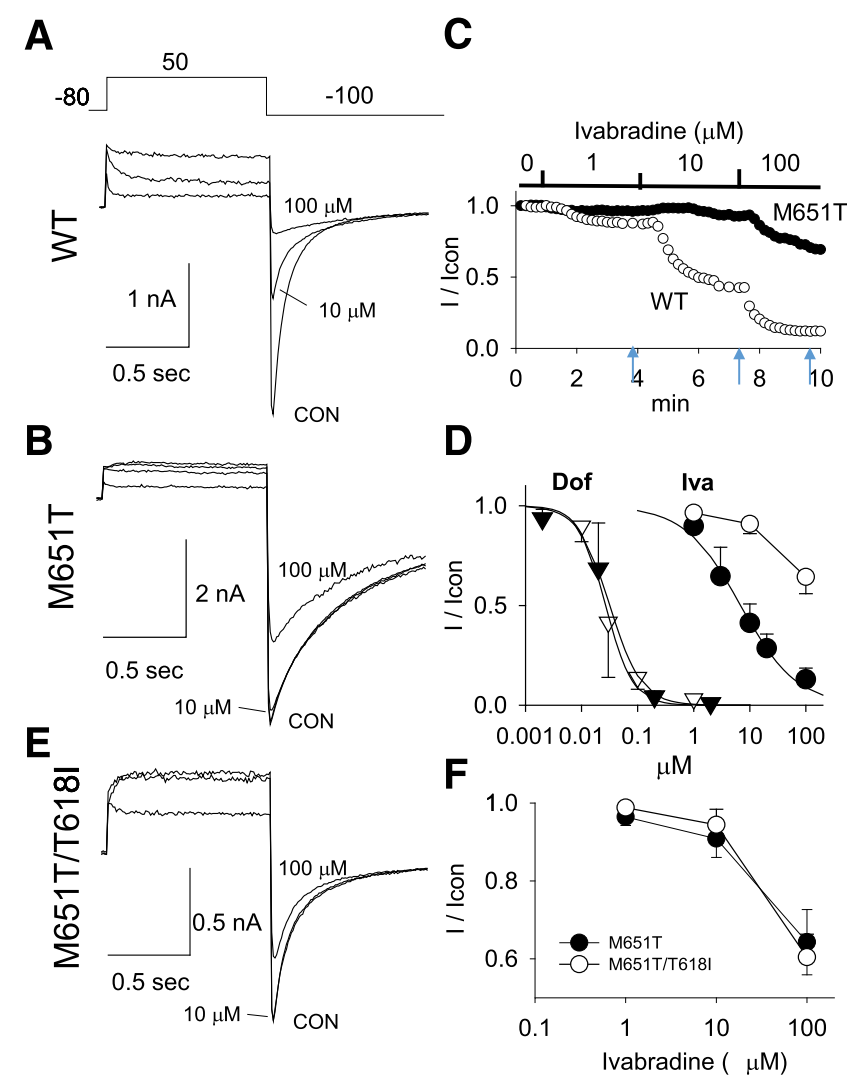

Fig. 2. Effect of ivabradine on WT-hERG1 (A) and M651T-hERG1 currents (B), respectively. (C) The representative time-courses of the WT and M651T current in response to application of various concentrations of ivabradine. The original current traces (times indicated with arrows) were shown in (A) and (B). (D) The dose-response curves of dofetilide (triangles) and ivabradine (circles) blockade of M651T-hERG1 (open symbols) and WT-hERG1 currents (solid symbols). For all experiments with ivabradine blocking M651T-hERG1, $n=3,6,7$ for concentrations of drugs 1, 10, and $100 \mu \mathrm{M}$, respectively. For ivabradine block of WT-hERG1, $n=5$ was used for every concentrations. The dose-response curves of dofetilide block of M651T-hERG1 were obtained with $n=5,7,5,10$ with concentrations of $0.01,0.03,0.1,1 \mu \mathrm{M}$. For dofetilide block of WT-hERG1, $n=1,3,2,2,5$ in the concentrations of $0.002,0.02,0.1,0.2,2 \mu \mathrm{M}$. (E) Effect of ivabradine on M651T/T618I hERG1 currents. (F) The dose-response curves of ivabradine blockade of M651T/T618I hERG1 in comparison with blockade of M651ThERG1. All experiments for M651T/T618I-hERG1 system were performed with $n=3$.

M651T modifies ivabradine-induced block by altering the deactivation time-course of the channel. To account for this covariable and to directly address whether the slowing of deactivation contributed to the shift in the concentrationresponse relationship to ivabradine, we created a double mutation, M651T/T618I. The rationale for its creation relates to a previous study wherein we reported that T618I accelerated deactivation kinetics. We empirically created the double mutation M651T/T618I to restore deactivation to WT values while retaining the key M651T substitution.

Mutations of Lipid-Facing Residue M651 Significantly Alters Thermodynamics and Kinetics of Ivabradine. Figure $2, \mathrm{~A}$ and $\mathrm{B}$, show raw data of the concentration-dependent ivabradine-induced block of WT hERG1 (Fig. 2A) in comparison with the M651T mutant (Fig. 2B). Figure 2C shows a representative time-course of block of $\mathrm{I}_{\text {hERG1 }}$ by ivabradine at various concentrations. $\mathrm{I}_{\text {herg1 }}$ designates the $\mathrm{I}_{\mathrm{kr}}$-like current elicited by transfecting the HEK cells with the hERG1 cDNA construct. Figure 2D shows the 
mean concentration-induced block comparing ivabradine (open circles for the WT, closed circles for the M651T) to dofetilide (open triangles, WT, and closed triangles, M651T; $n$ values are shown in the legend). At $10 \mu \mathrm{M}$ ivabradine suppressed $75 \%$ of the WT current, whereas at the same concentration, the drug blocked only $10 \%$ of the M651T current (Fig. 2D). The mean $\mathrm{IC}_{50}$ was $6.5 \pm 10 \mu \mathrm{M}$ for ivabradine in WT $(n=10)$, for M651T the $\mathrm{IC}_{50}$ was $>120 \pm 10 \mu \mathrm{M}(n=7)$, and beyond the drug's solubility limit. Note that to accurately measure $\mathrm{IC}_{50}$, a concentration higher than its solubility limit is essential. The impact of the lipid-facing residue M651 to ivabradine was also compared with dofetilide, a prototypical class III antiarrhythmic drug, to assess whether the mutant had a greater impact on response to ivabradine.

Interestingly, the M651T mutation had no impact on the concentration-response relationships to dofetilide. The mean $\mathrm{IC}_{50}$ concentration for dofetilide in WT was $41 \mathrm{nM}(n=4)$, whereas it was $27 \mathrm{nM}$ for the M651T mutation $(n=5)$. Thus, the $\mathrm{M} 651 \mathrm{~T}$ alters the concentration-response relation for ivabradine but not for dofetilide.

The stark contrast in the drug sensitivity for a lipid-facing mutation suggests that the two drugs are accessing the main intracavitary site of hERG1 via different pathways. The pKa values reported for the strongest basic chemical moiety in ivabradine (9.37) is similar but somewhat lower than of dofetilide (9.6). Nonetheless, the cationic form of the drug is a dominant form in the bulk aqueous solution at the physiologic $\mathrm{pH}$ values. However, the pKa values of weak-cationic compounds such as ivabradine are not the most accurate predictor of partitioning thermodynamics, since the equilibria between a neutral and a cationic form is a dynamic and environment-dependent process (DeMarco et al., 2018; Dickson et al., 2019).

To investigate thermodynamics of partitioning between aqueous phase and a model hydrophobic environment that mimics the membrane bilayer interior, we studied partitioning of ivabradine and dofetilide in an aqueous buffer and n-hexane. The thermodynamics of transfer for the two drugs showed significant differences. The partitioning free energy data summarized in Table 1 directly demonstrate that ivabradine has probabilities of partitioning nearly equal between aqueous buffer and a bulk hexane (Table 1). The free energy of transfer $\left(\Delta G_{t r}^{o}\right)$ between aqueous phase and a bulk hexane for $\mathrm{T}=313.15 \mathrm{~K}$ are $0.5 \pm 0.1$ and $8.4 \pm 0.2 \mathrm{~kJ} \mathrm{~mol}^{-1}$ for ivabradine and dofetilide, respectively. Therefore, partitioning data provide direct evidence that ivabradine is a lipophilic molecule, which partitions into the lipid compartment of the plasma membrane compared with dofetilide (Table 1). These findings are in excellent agreement with our previously published modeling studies (Lees-Miller et al., 2015).

Although M651T Slows Deactivation, Its Impact on Ivabradine-Induced Block Appears Independent of Deactivation. In comparison with WT, M651T mutation slows deactivation; the taus of WT hERG1 and the mutant were $160 \pm 40$ and $400 \pm 40$ milliseconds $(P<0.01)$, respectively. Figure $2, \mathrm{~A}, \mathrm{~B}$, and $\mathrm{E}$, show the raw deactivation time-course in response to ivabradine in WT (Fig. 2A) and M651T (Fig. 2B) and in a double mutation which rescues deactivation to the WT values (Fig. 2E). Figure $2 \mathrm{~B}$ shows the drug free time course of deactivation of the M651T mutation compared to the WT (Fig. 2A). The double mutant channel, M651T/T618I restored the deactivation time-course of the channel to values similar to WT hERG1 (compare Fig. 2, A, B, C, and E), both drug-free and with ivabradine. Even the double
TABLE 1

Mole fractions $\left(\mathrm{x}_{S}\right)$ and associated thermodynamic parameters for dofetilide and ivabradine hydrochloride partitioning in buffer-hexane system at $\mathrm{T}=298.15$ and $313.15 \mathrm{~K}, \mathrm{pH}=7.4$, and pressure $P=0.1 \mathrm{MP}$

\begin{tabular}{cccccc}
\hline & Temperature & Buffer & Hexane & $\log D_{H / B}$ & $\Delta G_{t r}^{o}$ \\
\hline \multirow{4}{*}{ Dofetilide } & $K$ & $x_{B} \cdot 10^{6}$ & $x_{H} \cdot 10^{6}$ & & $k J \cdot \mathrm{mol}^{-1}$ \\
& 313.15 & 5.38 & 0.215 & -1.40 & $8.4 \pm 0.2$ \\
Ivabradine & 298.15 & 5.34 & 0.258 & -1.32 & $7.9 \pm 0.2$ \\
& 313.15 & 3.55 & 2.93 & -0.08 & $0.5 \pm 0.1$ \\
& 298.15 & 3.69 & 1.67 & -0.34 & $2.0 \pm 0.1$ \\
\hline
\end{tabular}

mutant of the hERG1 channel that contains M651T still shifted the $\mathrm{IC}_{50}$ concentration-response of ivabradine by more than two orders of magnitude (Fig. 2F). The mean $\mathrm{IC}_{50}$ values of $\mathrm{M} 651 \mathrm{~T}$ were nearly identical to that of the double mutation M651T/T618I (Fig. 2F). These data indicate that decreased pharmacological sensitivity to ivabradine was not the result of slowing deactivation kinetics induced by the M651T mutation.

Impact of Other M651X Substitutions on IvabradineInduced Block. The relationship between baseline drugfree electrophysiological characteristics and $\mathrm{IC}_{50}$ values were evaluated by creating various substitutions at the M651 site (Supplemental Figs. 1-4). We sought to address whether there was a relationship between electrophysiological characteristics and $\mathrm{IC}_{50}$ response to ivabradine. We observed no significant correlation between drug-free voltage-dependence of activation $\left(V_{1 / 2}\right)$, deactivation kinetics, or voltage-dependence of C-type inactivation and the concentration-responsiveness to ivabradine. However, there was a modest correlation between the drug-free time-constant of recovery from inactivation and the mean $\mathrm{IC}_{50}$ values of ivabradine-induced block $\left(R^{2}=0.4\right)$. We next examined the effect of an $\mathrm{IC}_{50}$ concentration of ivabradine on ion currents elicited in various substitutions at the M651 site. The voltage-dependence of deactivation (during ivabradine treatment) and the kinetics of recovery from inactivation and their associated $\mathrm{IC}_{50}$ values were significant, but these correlations were quite modest. Although many substitutions at this lipid-facing residue (Supplemental Table 2) have significant impact on responsiveness to ivabradine, correlation between "the lipophilicity of the residues" and the mean $\mathrm{IC}_{50}$ values for ivabradine block was limited or even absent $\left(R^{2}<0.1-0.4\right)$. There were no apparent correlations between the $\mathrm{IC}_{50}$ for ivabradine binding or volume or solvent-accessible area of the residues in the 651 position (Supplemental Fig. 3). It is important to mention that in the absence of ivabradine, several of the M651X mutants exhibited altered gating behavior (Supplemental Figs. 3 and 4).

Therefore, ivabradine-induced block of hERG1 current could not be reduced to a simple drug binding in the vicinity of M651X. The drug action may be coupled to the gating process of the channel or altered accessibility to the intracellular cavity of the channel via some form of allosteric regulation. Essentially, drug-induced shifts in the time-course of recovery from inactivation appear to be a probable determinant of ivabradine potency. This coupling between presence of binding pockets, drug-channel interactions at the lipid-facing surface, and gating dynamics is often described in terms of a complex allosteric mechanism in which change in the topology of the binding pocket or inter-residue interactions modulates access or affinity for the substrate binding pocket (Gordon and Zagotta, 1995). 
A

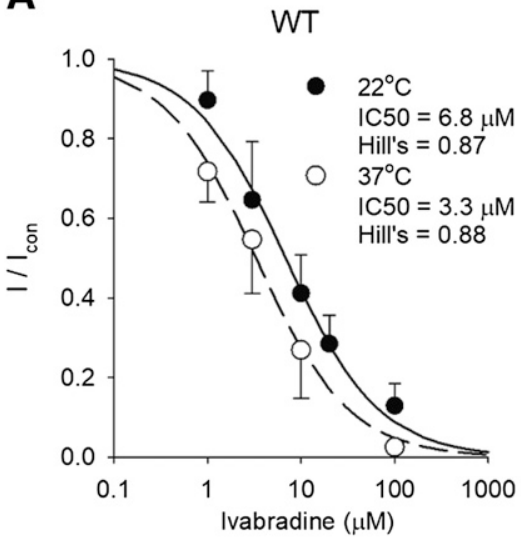

B

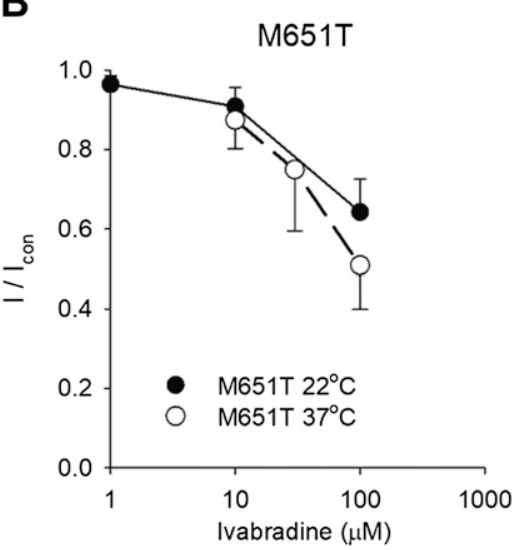

Fig. 3. The temperature dependence of the concentrationresponse curves for ivabradine blockade of WT-hERG1 (panel A) and M651T-hERG1 (panel B) currents at $\mathrm{T}=22^{\circ} \mathrm{C}$ and $37^{\circ} \mathrm{C}$ shown as closed and open circles, respectively. In the WT-hERG1 experiments at $\mathrm{T}=22^{\circ} \mathrm{C}, n$ were $6,5,5,5,5$ for concentrations of $1,3,10,20,100 \mu \mathrm{M}$ of ivabradine. $\mathrm{T}=37^{\circ} \mathrm{C}$, $n=8,7,3,4$ for drug concentrations of $1,3,10,100 \mu \mathrm{M}$ respectively. In the $\mathrm{M} 651 \mathrm{~T}$ construct at $\mathrm{T}=22^{\circ} \mathrm{C}, n=3,6,7$ for experiments performed with $1,10,100 \mu \mathrm{M}$, respectively. At $\mathrm{T}=37^{\circ} \mathrm{C} n=4,3,5$ for $10,30,100 \mu \mathrm{M}$, respectively.
Temperature Dependence of Ivabradine Blockade. The measured $\mathrm{IC}_{50}$ values for ivabradine in WT hERG1 differ somewhat between various laboratories. We note that our originally published $\mathrm{IC}_{50}$ value of $6.8 \mu \mathrm{M}$ differs from that of Melgari et al. (2015). Their measurements were reported at $37^{\circ} \mathrm{C}$, whereas our measurements were made at $22^{\circ} \mathrm{C}$. Accordingly, to address whether experimental conditions accounted for some of these differences in measured $\mathrm{IC}_{50}$ values we directly compared $\mathrm{IC}_{50}$ values of ivabradine at $37^{\circ} \mathrm{C}$ versus $22^{\circ} \mathrm{C}$. Our measured wild-type $\mathrm{IC}_{50}$ value at $37^{\circ} \mathrm{C}$ of $3.3 \mu \mathrm{M}$ is relatively close to the values of $2.07 \mu \mathrm{M}$ for hERG1 and $3.31 \mu \mathrm{M}$ for WT-hERG1 1a/1b isoform reported by Melgari et al. (2015). Most importantly, we further re-established that the impact of M651T was not significantly altered by increasing the temperature to $37^{\circ} \mathrm{C}$ (Fig 3B). In fact, higher temperature may induce more potent block by ivabradine. The measured $\mathrm{IC}_{50}$ of ivabradine decreased from 6.8 to $3.3 \mu \mathrm{M}$ with an increase in temperature, paralleling more favorable partitioning of ivabradine into the hexane at a higher temperature (Fig. 3; Table 1). Given that ivabradine is a highly lipophilic compound, the drug precipitates at higher concentrations as in these experiments, thereby making it impossible to calculate an exceedingly accurate $\mathrm{IC}_{50}$ value for ivabradine block of M651T, because the solubility of ivabradine prevents evaluations at concentrations $>100 \mu \mathrm{M}$.

Interplay Between Aromatic Cassette (Y652, F656, and F557) Dynamics and Ivabradine-Induced Block. A vast number of studies describing molecular determinants of high- to mid-affinity blockade have emphasized an important role played by aromatic residues in the pore-domain cavity of the channel (Y652 and F656) (Duff et al., 1995; Ficker et al., 1998; Lees-Miller et al., 2000, 2015; Mitcheson et al., 2000; Perry et al., 2010; Saxena et al., 2016). These residues are pivotal determinants of class III drug-induced block of $\mathrm{I}_{\mathrm{hERG}}$; thus a range of double mutations were created to enhance our understanding of the interplay between those key residues in the pore domain and the lipid-facing residue M651 (Ficker et al., 1998; Lees-Miller et al., 2000; Mitcheson et al., 2000; Perry et al., 2010; Saxena et al., 2016). Figure 4 shows the pharmacological responses of ivabradine to the single and double mutations. Figure 4, A-C, show the effects of adding the M651T mutation to F656C, Y652, or S620T. S620T is a mutant with impaired C-type inactivation phenotype (Herzberg et al., 1998; Perry et al., 2007) that also has a major impact on channel-induced activation or block by small molecules (Ficker et al., 1998; Herzberg et al., 1998; Wu et al., 2014, 2015). We previously reported that the single mutation S620T decreases the pharmacologic responsiveness of $\mathrm{I}_{\text {hERG1 }}$ to ivabradine (Lees-Miller et al. 2015).

A large concentration of ivabradine $(100 \mu \mathrm{M})$ only partially blocks the F656C (72\% block), Y652A (30\%), M651T (30\%), and S620T currents (32\%), but almost no block was observed at the same concentration of ivabradine with either the M651T/ F656C (<1\% block), M651T/S620T (1\% block), or M651/Y652A (12\%) compared with their respective individual mutations as seen in Fig. 4 ( $P$ and $n$ values are shown in the legend and statistical analysis of the data are provided in the Supplemental Table 1). The analysis of electrophysiological recordings in Fig. 4 show no additive or synergistic interplay between F656 and Y652 (Fig. 4E). These data indicate that the M651 site substantially modifies the impact of known aromatic mutations in the distal S6. Mutations of F557L and M651T, both, impaired ivabradine-induced block, but surprisingly the block of the double mutant F557L/M651T was similar to the WT values (Fig. 4D and the raw data are shown in Fig. 5A,B). Panel A shows the raw data before and after ivabradine addition. Panel B shows the mean IC50 concentration-reponse plots. Panel $\mathrm{C}$ shows the washout. These data indicate F557 in the S5 and M651 in the distal S6 negatively interplay to rescue pharmacologic response of ivabradine. However, no significant negative or positive interplay is observed with single or double mutations of F557L and S620T (Fig. 4F). In review, the M651 residue interplays positively with many other key residues that are structural determinants of ivabradine-induced block. In contrast, M651T interplays negatively with F557L (Fig. 5B). These data suggest a presence of a complex allosteric interaction of ivabradine with residues in hERG1-a novel mechanism established in this work.

\section{Discussion}

State-Dependence in the Ivabradine Blockade of hERG1 Currents. One of the most important determinants of hERG1 block-associated proarrhythmia is the statedependent kinetics of drug interactions with the channel (Di Veroli et al., 2013a, 2013b; Hill et al., 2014). The landmark feature of hERG1 channel kinetics is the rapid C-type inactivation (Sanguinetti et al., 1995; Schönherr and Heinemann, 1996; Spector et al., 1996). A number of mutations significantly 
A

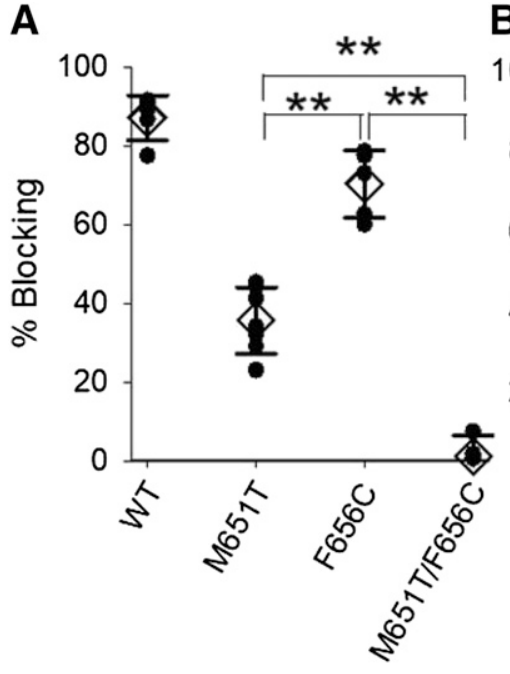

B
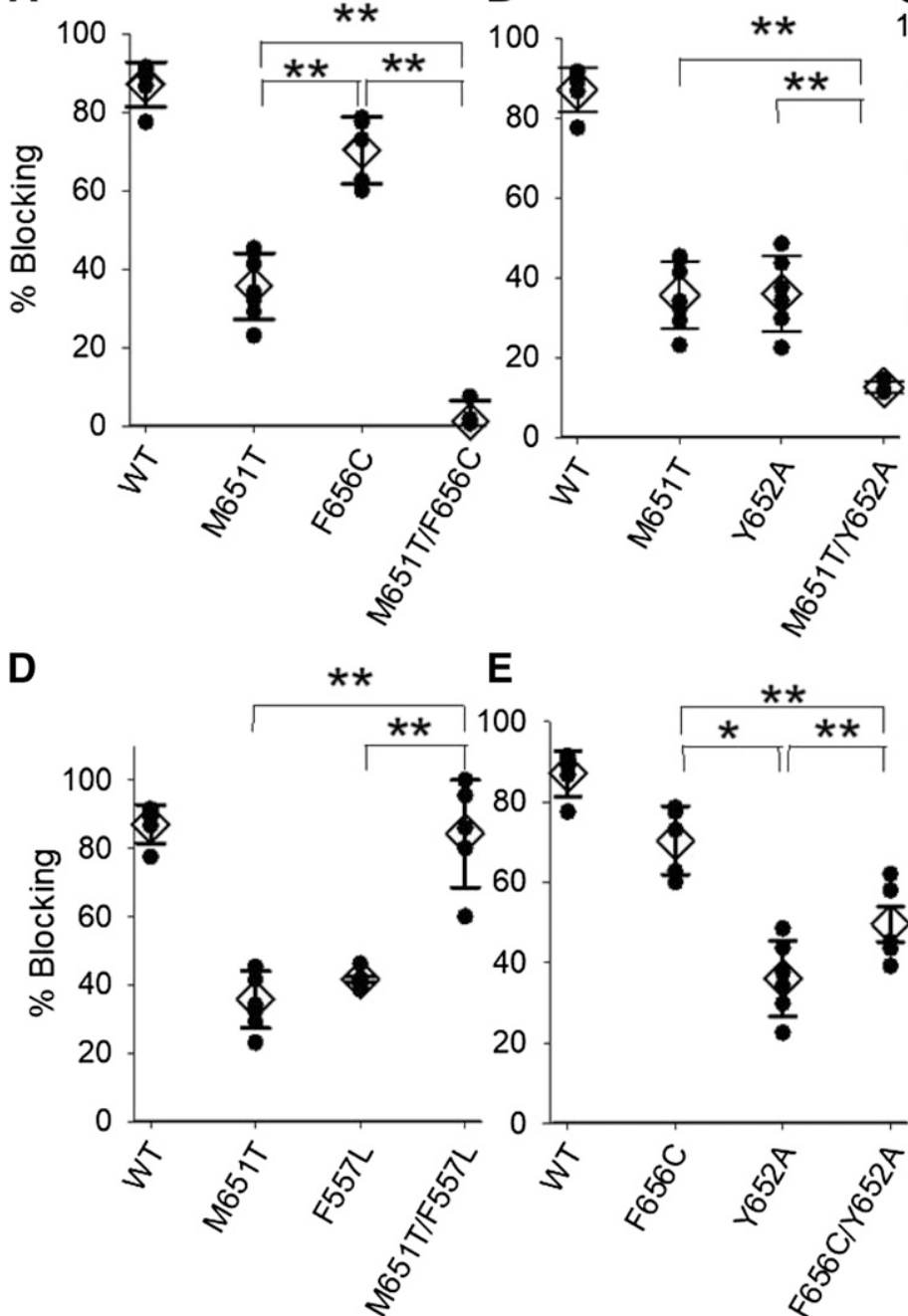

C

E

F

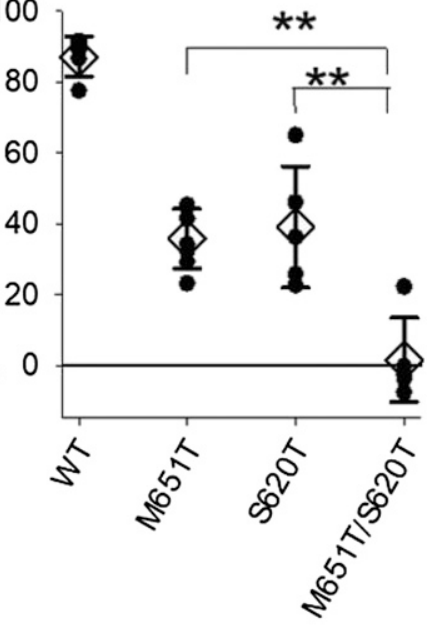

Fig. 4. Analysis of concomitant interactions of double mutations in blocking effects of saturating concentration of ivabradine (100 $\mu \mathrm{M})$. Blocking effect of (A) M651T/F656C, (B) M651T/Y652A, (C) M651T/S620T, (D) M651T/F557L, (E) F656C/Y652A, (F) S620T/F557L, and their corresponding single mutations are shown. The following number of experiments was used: $N=5,7,5,4$ in order of in (A); $5,7,6,3$ in (B); $5,7,5,5$ in $(\mathrm{C}) ; 5,7,5,5$ in (D); 5, 5, 6, 5 in (E); 5, 5, 5, 4 in (F). ${ }^{*} P<0.05 ;{ }^{*} P<0.01$. One way ANOVA analysis was used. The precise statistic $P$ values are provided in the Supplemental Table 1. WT was used as reference and was not included in the statistical analysis.

alter or shift the voltage-dependence of C-type inactivation, including the S620T mutant. Therefore, the contribution of inactivation of M651T mutation on ivabradine-induced block was assessed by comparing the extent of block in M651T, S620T, and the double mutant M651T/S620T. By combining a noninactivating pore (S620T) with the M651T mutation, drug block was virtually eliminated. These data indicate that both the process of C-type inactivation and the M651 site in the distal S6 interact to produce at least additive impairment of ivabradine-induced block. Collectively, the results described for mutations in the positions F557, M651, Y652, and F656 indicate that drug blockade depends on the coupling between conformational dynamics of the residues in the pore domain and on a mechanism that involves repacking of the lipid- and/ or pore-facing residues. How are state-dependent conformational dynamics of F557, M651, Y652, and F656 coupled to drug access/binding to a pocket? To understand dynamics of these residues we performed 1.2- to 2.5-microsecond all-atom molecular dynamics simulations in an explicit water/membrane system for selected mutants.
Orientations of F557 and F656 are State-Dependent Properties: From Pore-Lining to Lipid-Facing. The availability of new cryo-EM structures for hERG1 and hEAG1 channels in combination with recent developments in MD simulations allowed us to investigate structural dynamics of the open and closed states along with mutants of interest, hence allowing us to test directly the hypothesis presented above. In the previous and widely accepted mechanistic models, the aromatic residues (Y652 and F656) were postulated to face the permeation pathway and coordinate drugs bound to the intracellular cavity (Chen et al., 2002; Perry et al., 2010). Various structural models with bacterial $\mathrm{K}^{+}$channels or Shaker-family of $\mathrm{K}^{+}$channels as their bases emphasized the importance of direct interactions between drugs bound in the water-filled cavity with F656 and/or Y652 (Perry et al., 2010; Wacker et al., 2017). The recent progress in cryo-EM technologies allowed us to model open and closed states of hERG1 channel. Although the model based on hEAG1 represents a closed pore, a cryo-EM structure for hERG1 corresponds to the open state. Importantly, spatial 
A

A

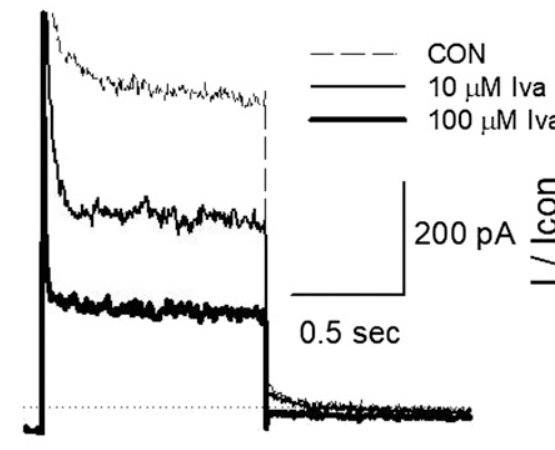

Ivabradine

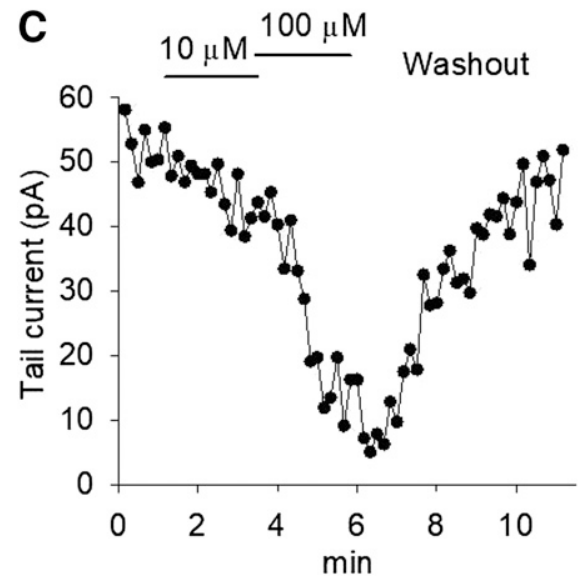

$\mathbf{B}_{1}$

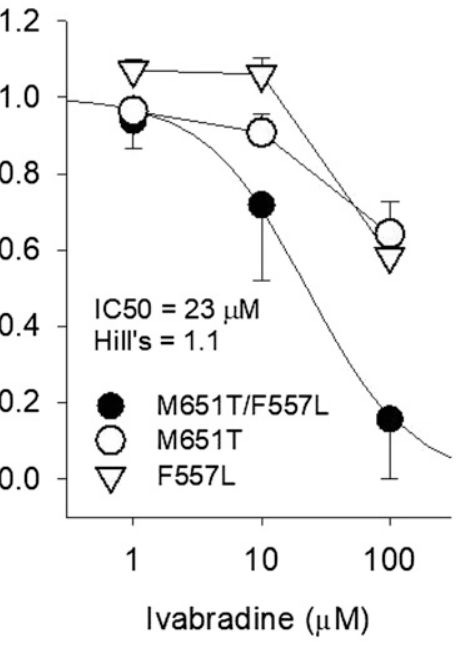

Fig. 5. Current traces (A) and time-course (C) of ivabradine on M651T/F557L and their single mutations. (C) The concentration-response curves. The smooth curve was fitted to the Hill equation. Fitting of M651T and F557L was not possible. (E) The following numbers of experiments were performed: $N=3,4$, and 5 in F557L; $n=3,6$, and 7 in M651T; $n=4,5$, and 5 in M651T/F557L in concentrations of 1,10 , and $100 \mathrm{mM}$, the legend is shown on the figure. (D) Patch-clamp protocol

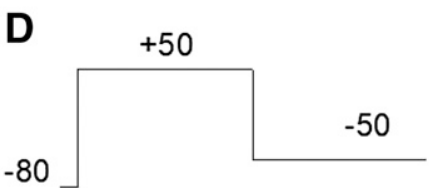

orientation of F656 and F557 side-chains display remarkable state dependence. As predicted by a majority of structural modeling studies, the F656 is pointing toward the inside of the cavity in the closed-state model (Wang et al., 2016; Wacker et al., 2017)). All-atom MD simulations indicate that F656 can rotate away from the permeation pathway and in the open-state model flickers between the intracavitary and the lipid-facing orientations (Fig. 6, A-C). In the open state, the position of $\mathrm{F} 656$ aromatic ring is in proximity to F557 and is close enough to form stable $\pi-\pi$ stacking interactions at the lipophilic entry pathway.

To assess properties of the putative lipid-entry pathways and the impact of various mutations on the dynamics of the F557-M651X-F656 motif, we performed topological analysis of the production portion of MD simulations (for traces, see Supplemental Figs. 5 and 6) using the MOLEonline pathway analyzer (Berka et al., 2012). Two potential entry pathways were mapped from analysis of MD trajectories. Both pathways are defined by conformational states of F656 and F557 residues and illustrated in Fig. 6A. We analyzed the conformational dynamics of the F557-F656 pair to gain additional insight regarding the flexibility of a tentative lipid-facing binding site and its impact on the accessible volume required for drug diffusion into the primary intracavitary site (Supplemental Fig. 5). The conformational space of F557F656 interacting pair and the impact of M651T mutation in different states of hERG1 is illustrated in Fig. 6, B-D. The direct interactions between F656 and F557 appear to be only quasi-stable (flickering state) in the WT hERG1 (Fig. 6, B, C, $\mathrm{D}, \mathrm{E})$. These interactions establish a large accessible volume for drug binding on the lipophilic site of the intracellular cavity. The interaction of the pair is state-dependent, which may directly support the previously postulated role of F656 in the state-dependent drug blockade of hERG1channel (Chen et al., 2002). Therefore, it is tempting to assign the F656-F557 pair a pivotal role in state-dependent gate control of ivabradine diffusion from the lipid bilayer to the main binding site in the intracellular cavity. The additional analysis of M651T mutant shows that the conformational flexibility of the F656 and F557 pair is significantly impeded with the mutant (Fig. 6C, D, E). Evidently, the M651T mutation significantly stabilizes the pair (F656 and F557) by decreasing the F656 "flickering" frequency (Fig. 6, B and C). Other mutations affecting conformational flexibility of F656 or F557 (Supplemental Figs. 7-14) are also associated with inhibition of hERG1 blockade by ivabradine. The structural states of F656 appear to be important modulators of high-affinity binding for major hERG1 blockers (Supplemental Fig. 14; Supplemental Table 4).

In review, the state-dependent orientation of F656 appears to be an important determinant of a putative lipophilic entry pathway explored by some of the hERG1 blockers, including ivabradine. The all-atom MD-refined models of hERG1 channel in its open and closed states demonstrate that F656 can rotate away from the intracellular cavity toward the lipid bilayer and form hydrophobic interactions with F557 and M651 residues (Fig. 1). Interestingly, the conformational flexibility of F656 (it can be directly modulated by mutations in the position M651 or F557) is in excellent correlation with findings observed experimentally in ivabradine blockade to WT and mutant forms of hERG1. 
A
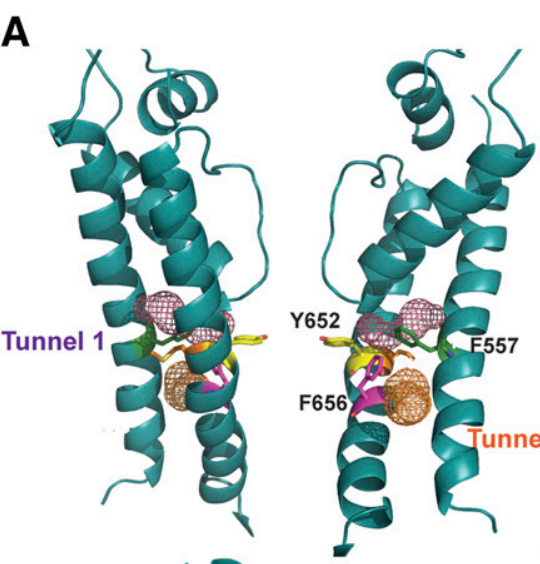

B

C
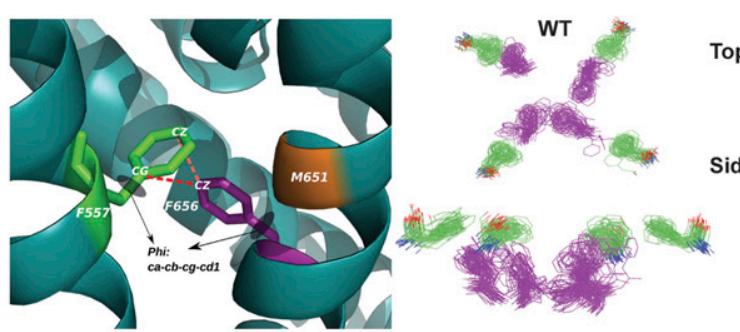

Top

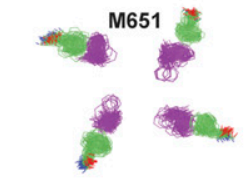

D
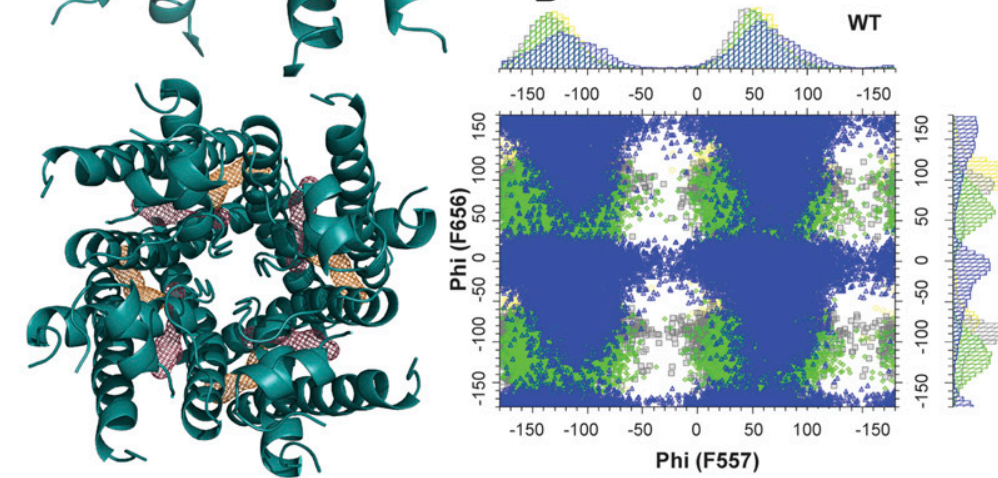

E
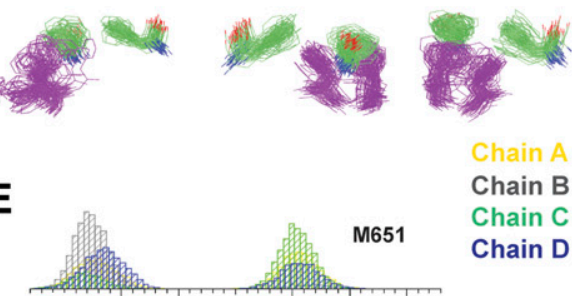

$\begin{array}{lllllll}-150 & -100 & -50 & 0 & 50 & 100 & -150\end{array}$

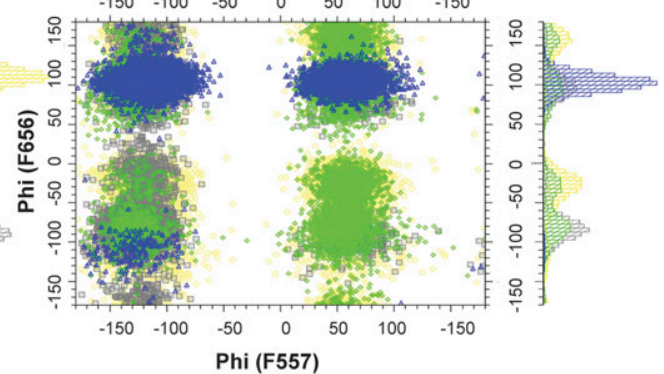

Fig. 6. Impact of the M651T mutation on the conformational dynamics of aromatic cassette in WT-hERG1 and M657T-hERG1 systems. The dihedral distributions and accessibility mapping were obtained from the last 750 nanoseconds of equilibrium all-atom MD simulations. (A) Side view of two subunits and top view of the pore domain showing relevant access pathways mapped by the MOLEonline tool (see Supplemental Information for details). Selected residues are shown: F557 (green), F656 (magenta), M651 (orange), and Y652 (yellow). (B) Key distances and torsional angles involving F557 (green sticks) and F656 (magenta sticks). Position of residues M651 in the S6 helix are colored in orange. (C) Top and side views of the superimposed positions of F557 (green) and F656 (magenta) for WT-hERG1 (left) and M651T-hERG1 (right). (D and E) one-dimensional and two-dimensional distribution maps for torsional angles in each subunit of WT-hERG1 (D) and M651T (E). One-dimensional distributions are shown for F557 (top panel) and F656 (side panel), respectively.

Induced-Fit Docking and MD-Ensemble Docking Supports Coexistence of Binding Sites in the Pore Cavity and Pore-Lipid Interface. The extensive sampling of various conformational states of WT and mutant forms of hERG1 channel in different states achieved with Anton 2 platform allowed us to map tentative binding sites for ivabradine. Potential binding sites around the mutated residues were explored through docking of ivabradine to different sections of the protein using hERG1 in different states, also incorporating ensemble docking, where clusterrepresentatives from all-atom MD simulations were used. Two main binding sites, "lipophilic site" and "internal cavity site," were further analyzed and compared for WT hERG1 and mutants. The stable and populated binding sites from Induced-Fit Docking are shown in Fig. 7, and a summary of docking energetics for all studied systems are shown in Tables 2 and 3 . There were no poses found for ivabradine binding (either neutral of cationic form) to the main internal cavity of the hERG1 for the closed state of the receptor (Table 2). For the closed state of hERG1, ivabradine shows stable binding to the pocket centered at M651, in agreement with the previously proposed lipophilic access site (Lees-Miller et al., 2015) (Table 2). In contrast to the closed state of the channel, the docking to the open state revealed three stable binding modes. Ivabradine was favorably bound to the main pocket in the intracellular cavity, to an area around a fenestration window (in-between $\alpha$-helices of S6), or to a lipid-facing binding pocket (Fig. 6A). The IFD docking performed to an open state of the hERG1 channel or the MD simulations to an ensemble of open structures show similar binding affinities for ivabradine (Table 3). The results of IFD docking qualitatively agrees with the experimental data (Table 2). At the same time, results from the MD-ensemble docking inherently have large uncertainties in computed binding affinities, rendering comparisons between mutants rather difficult (Table 3). However, the ensemble-based docking simulations allow for better quantification and comparison of relative populations found in each of the three binding sites. The population analysis is essential for understanding the binding processes that involve large and flexible ligands such as ivabradine (Zhao et al., 2010; Shoichet and Kobilka, 2012). The relative populations of binding poses found for identified sites are shown in Fig. 7 and Table 3. Comparing number of poses from the docking simulations, ivabradine preferentially binds to the lipophilic site or the internal cavity of the WT hERG1. However, a substantial number of poses are also found near a potential fenestration window or the "access pathway" (Fig. 7A; Supplemental Fig. 6).

For additional insights, we performed docking simulations on selected mutant systems, specifically M651T, F557L, and F557L/M651T hERG1 mutants. It was found that in both M651T and F557L mutants, ivabradine showed a preferential binding to the intra-cellular cavity of the channel and a very small occupancy (number of poses) in the lipophilic binding pocket. In conclusion, ensemble docking for both mutants M651T and F557L indicate disruption of the lipophilic access site owing to the repacking of this binding pocket caused by the mutations. The mutations in 651 and 557 reduced relative 
A
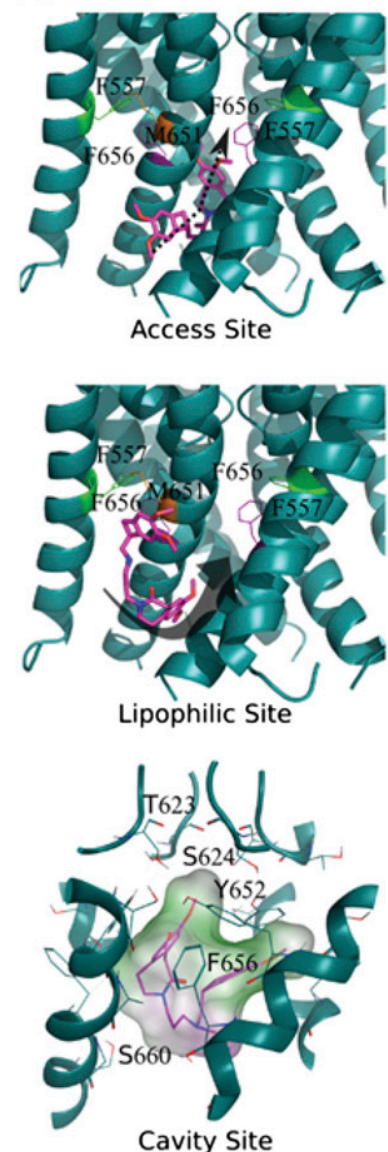

B

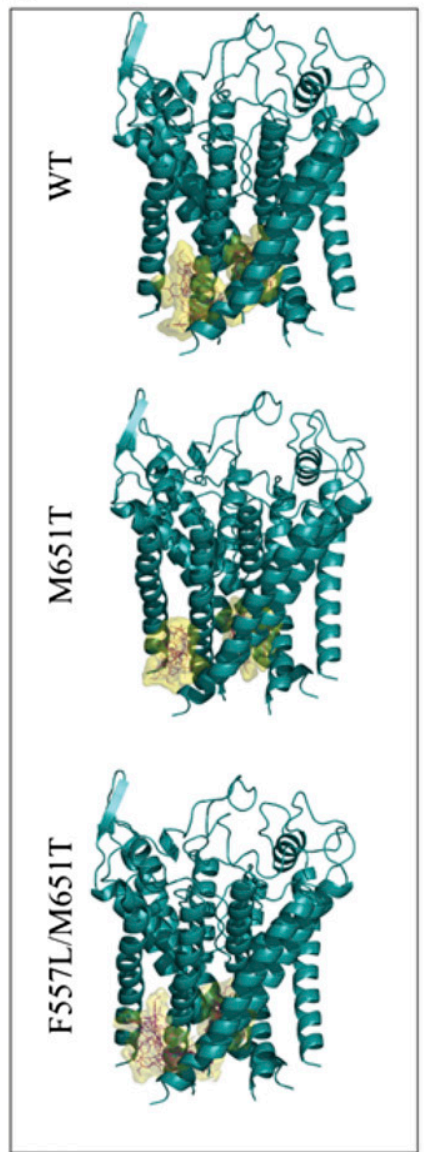

C

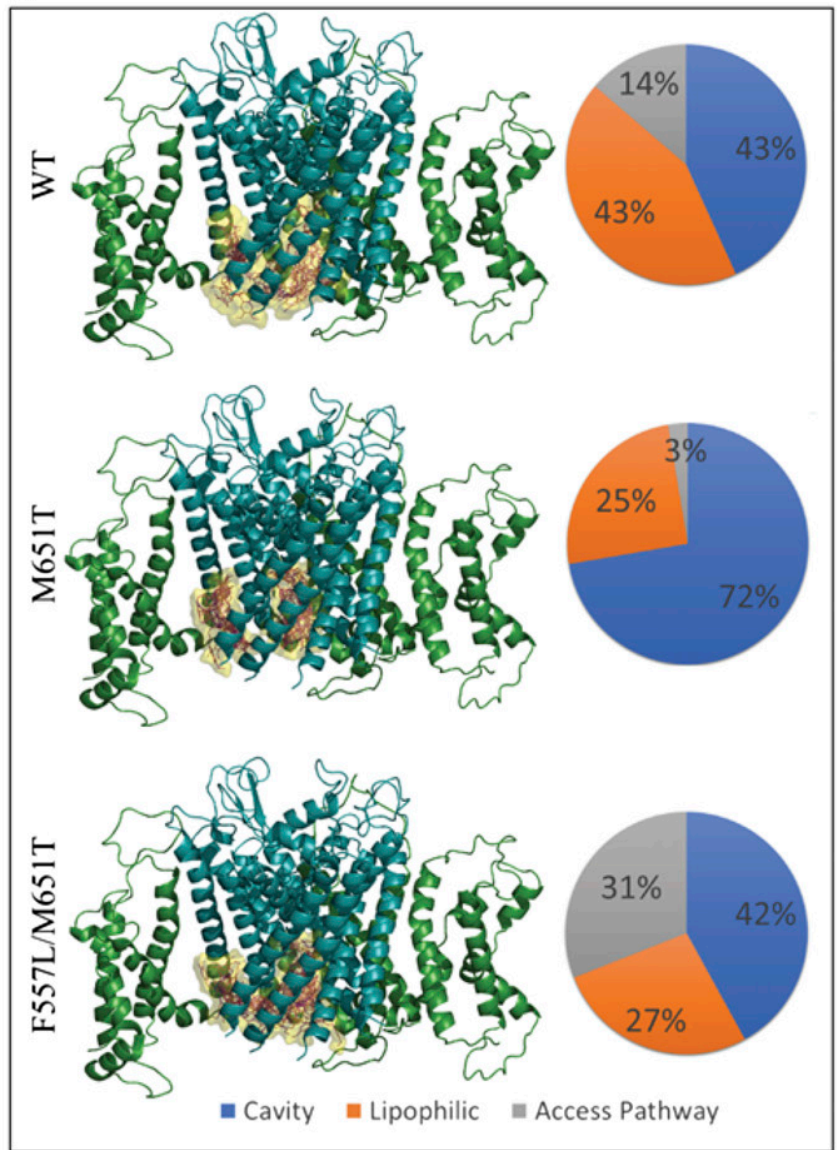

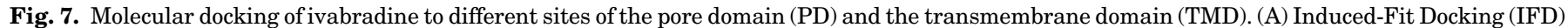

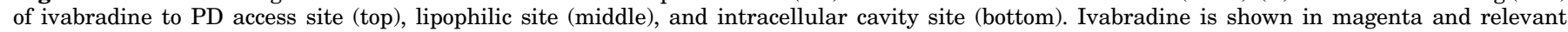

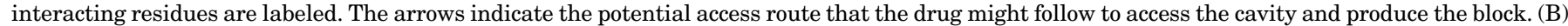

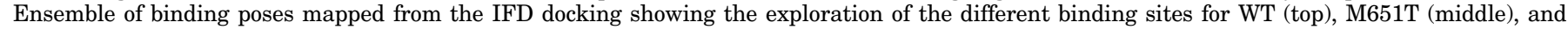

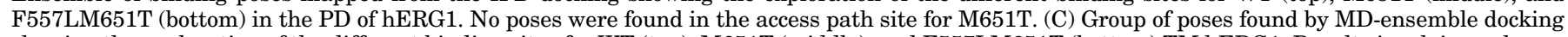

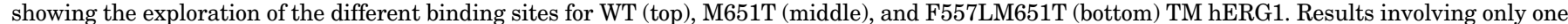

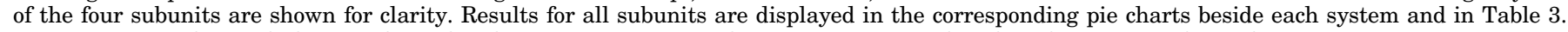
In agreement with IDF docking performed with cryo-EM structure, almost no poses were found on the access path site for M651T mutant.

population of binding poses in the binding pocket near a potential fenestration window (access path to the internal cavity path) by 2 -fold compared with the WT system. These findings are in excellent agreement with the electrophysiological data presented herein. The data combined for the double mutant M651T/F557L indicate almost WT-like binding affinities in all three mapped binding sites with modest increase in binding affinity for intracellular cavity- and the access binding sites (Fig. 7C). Therefore, the double mutant is expected to have WT-like binding properties in the open state of the channel. We conclude that the flexible nature of F656 sidechain, which can rotate toward the lipid-facing side and the internal cavity of the channel seems to be a determining factor in ivabradine-induced block. MD simulations have also shown that the double mutant F557L/M651T restores the WT-like dynamic of F656 flickering, therefore re-establishing drug occupancy in the lipophilic access site for ivabradine. Subsequent ivabradine docking to WT hERG1 and selected mutants (M651T, F557L, and F557L/M651T) indicated that the lipophilic site is accessible to both open and closed states of the channel. However, the closed state of the channel contains no binding pockets for ivabradine in the intracellular cavity and the "access" binding site near the fenestration window. The simulation data suggest that the structural rearrangements of this binding pocket during the channel's activation may play a role in ivabradine binding to the lipophilic site and subsequent access to the entry site and ultimately into the intracellular cavity of the channel. Molecular docking simulations

TABLE 2

Binding affinities of ivabradine docking to hERG1 pore-domain models Binding affinities of the best pose for WT and mutants are shown. Docking boxes were defined around the centroid of residues F557 and M651.

\begin{tabular}{|c|c|c|c|c|}
\hline \multirow{2}{*}{ PD System } & \multicolumn{2}{|c|}{$\begin{array}{c}\text { Closed hERG1Model } \\
20 \AA \text { Box, BE }\end{array}$} & \multicolumn{2}{|c|}{$\begin{array}{c}\text { Open hERG1 Cryo-EM } \\
\text { Model } 15 \AA \text { Box, BE }\end{array}$} \\
\hline & Lipophilic & Cavity & Lipophilic & Cavity \\
\hline & \multicolumn{2}{|c|}{$\mathrm{kcal} / \mathrm{mol}$} & \multicolumn{2}{|c|}{$\mathrm{kcal} / \mathrm{mol}$} \\
\hline $\mathrm{WT}^{a}$ & -5.45 & No Poses & -7.36 & -8.59 \\
\hline F557L/M651T ${ }^{a}$ & -5.05 & & -6.76 & -8.89 \\
\hline M651Ts & -4.84 & & -4.56 & -7.95 \\
\hline F557L & -4.96 & & -5.44 & -7.84 \\
\hline
\end{tabular}

${ }^{a}$ Poses in the access path to internal cavity were found, see Fig. 4, Supplemental Fig. 3, and Supplemental Table 3 . 
TABLE 3

Ivabradine affinity to hERG1 transmembrane domain from GlideXP docking to the selected MD frames of WT and mutant hERG1

Poses were clustered into three binding sites: cavity, lipophilic, and "access path." Average energy considering all four subunits is presented as kilocalories per mole as well as the percentage of poses found within the binding site.

\begin{tabular}{lccc}
\hline \multirow{2}{*}{ TM System } & \multicolumn{3}{c}{ hERG1 Cryo-EM Model } \\
\cline { 2 - 4 } & Cavity & Lipophilic & Access Path \\
\hline & $k c a l / m o l$ & $k c a l / m o l$ & $k c a l / m o l$ \\
WT & $-5.22 \pm 1.45(43.2 \%)$ & $-4.72 \pm 0.93(43.1 \%)$ & $-4.42 \pm 0.93(13.7 \%)$ \\
M651T & $-5.71 \pm 1.31(72.1 \%)$ & $-4.58 \pm 0.74(25.3 \%)$ & $-4.19 \pm 1.40(2.6 \%)$ \\
F557L & $-5.57 \pm 1.27(67.2 \%)$ & $-4.75 \pm 0.89(26.6 \%)$ & $-4.27 \pm 0.72(6.1 \%)$ \\
F557L/M651T & $-4.69 \pm 1.10(41.8 \%)$ & $-4.76 \pm 0.99(27.3 \%)$ & $-5.00 \pm 1.08(30.9 \%)$ \\
\hline
\end{tabular}

performed for the cationic form of ivabradine targeting primary site in the intracellular cavity show comparable binding free energies (to the lipophilic site) and also underline the crucial role of F656 dynamics (Supplemental Table 4) coupled to the complex interactions with F557 and M651 residues.

\section{Conclusions}

In summary, we show that the pore-lipid interface mutation (M651T) significantly impairs ivabradine-induced block of the hERG1 current but does not alter dofetilide-induced block. Thus the impact of M651T appears to be specific to ivabradine and emphasizes the underlying important role of a new lipophilic access pathway. The structural mechanisms of the observed lipophilic binding of ivabradine were discerned from a combination of microseconds-long MD and traditional IFD/MD-ensemble docking simulations. The modeling data emphasize the role of M651 as an allosteric modulator of statedependent hydrophobic interaction between F557 and F656, both of which are well known determinants of ivabradineinduced block. The F557 residue was shown to interact with F656 by forming $\pi-\pi$ stacking interactions, an interaction that is disrupted by M651. The overall process of M651 tugging on F656 results in a mobile and flickering F656, which flickers to face the lipid-facing side and the internal cavity. Whereas $\mathrm{M} 651 \mathrm{~T}$ mutant is unable to interact with $\mathrm{F} 656$, it enhances F656 and F557 $\pi-\pi$ stacking interactions, resulting in a rigid F656. Thus, M651 controls the quasi-flickering state of F656 and shapes the topology of the binding pocket by controlling orientation of the F656/F557 hydrophobic cassette. MD simulations provided direct evidence that the mutations at these two positions (F557 and M651) cause changes in the topology in the vicinity of the proposed access and/or lipophilic binding sites by altering F656 flexibility and rotations, thereby impacting the number of binding poses and hence limiting accessibility to the main binding pocket in the intracellular cavity of the open-hERG1. We conclude that in WT-hERG1 channel F656, M651, F557 residues act as a dynamic gate that controls the pathway of drug access at the lipid-facing domain into the intracellular cavity of the channel. The experimental data provided firm evidence that the extent of ivabradine blockade recorded for F557L/M651T mutant with WT-like conformational dynamics was essentially similar to the WT hERG1.

\section{Acknowledgments}

We thank Dr. Marcela Madrid and Dr. Philip Blood of Pittsburgh Supercomputing Center for their assistance with running molecular dynamics simulations on Anton 2 platform. We express our gratitude to Martin Karplus and CHARMM project for waiving license fees for the CHARMM program package used for analysis of simulation data.

\section{Authorship Contributions}

Participated in research design: Perissinotti, Guo, Lees-Miller, Noskov, Duff.

Conducted experiments: Perissinotti, Guo, Kudaibergenova, Ol'khovich, Sharapova, Perlovich.

Contributed new reagents or analytic tools: Guo, Muruve, Gerull, Noskov, Duff.

Performed data analysis: Perissinotti, Guo, Kudaibergenova, Noskov, Duff.

Wrote or contributed to the writing of the manuscript: Perissinotti, Guo, Kudaibergenova, Muruve, Gerull, Noskov, Duff.

\section{References}

Abi-Gerges N, Holkham H, Jones EM, Pollard CE, Valentin JP, and Robertson GA (2011) hERG subunit composition determines differential drug sensitivity. Br J Pharmacol 164 (2b):419-432.

Bender BJ, Cisneros A III, Duran AM, Finn JA, Fu D, Lokits AD, Mueller BK, Sangha AK, Sauer MF, Sevy AM, et al. (2016) Protocols for molecular modeling with Rosetta3 and RosettaScripts. Biochemistry 55:4748-4763.

Berka K, Hanák O, Sehnal D, Banás P, Navrátilová V, Jaiswal D, Ionescu CM, Svobodová Vareková R, Koca J, and Otyepka M (2012) MOLEonline 2.0: interactive web-based analysis of biomacromolecular channels. Nucleic Acids Res 40(Web Server issue):W222-W227.

Best RB, Zhu X, Shim J, Lopes PE, Mittal J, Feig M, and Mackerell AD Jr (2012) Optimization of the additive CHARMM all-atom protein force field targeting improved sampling of the backbone phi, psi and side-chain chi(1) and chi(2) dihedral angles. J Chem Theory Comput 8(9):3257-3273.

Blokhina SV, Sharapova AV, Ol'khovich MV, Volkova TV, and Perlovich GL (2016) Solubility, lipophilicity and membrane permeability of some fluoroquinolone antimicrobials. Eur J Pharm Sci 93:29-37.

Brooks BR, Brooks CL III, Mackerell AD Jr, Nilsson L, Petrella RJ, Roux B, Won Y, Archontis G, Bartels C, Boresch S, et al. (2009) CHARMM: the biomolecular simulation program. J Comput Chem 30:1545-1614.

Chen J, Seebohm G, and Sanguinetti MC (2002) Position of aromatic residues in the S6 domain, not inactivation, dictates cisapride sensitivity of HERG and eag potassium channels. Proc Natl Acad Sci USA 99:12461-12466.

Chiamvimonvat N, Chen-Izu Y, Clancy CE, Deschenes I, Dobrev D, Heijman J, Izu L, Qu Z, Ripplinger CM, Vandenberg JI, et al. (2017) Potassium currents in the heart: functional roles in repolarization, arrhythmia and therapeutics. $J$ Physiol 595: 2229-2252.

Compton SJ, Lux RL, Ramsey MR, Strelich KR, Sanguinetti MC, Green LS, Keating MT, and Mason JW (1996) Genetically defined therapy of inherited long-QT syndrome. Correction of abnormal repolarization by potassium. Circulation 94: 1018-1022.

DeMarco KR, Bekker S, Clancy CE, Noskov SY, and Vorobyov I (2018) Digging into lipid membrane permeation for cardiac ion channel blocker d-sotalol with all-atom simulations. Front Pharmacol 9:26.

Dickson CJ, Hornak V, Bednarczyk D, and Duca JS (2019) Using membrane partitioning simulations to predict permeability of forty-nine drug-like molecules. J Chem Inf Model 59:236-244.

Di Veroli GY, Davies MR, Zhang H, Abi-Gerges N, and Boyett MR (2013a) Highthroughput screening of drug-binding dynamics to HERG improves early drug safety assessment. Am J Physiol Heart Circ Physiol 304:H104-H117.

Di Veroli GY, Davies MR, Zhang H, Abi-Gerges N, and Boyett MR (2013b) hERG inhibitors with similar potency but different binding kinetics do not pose the same proarrhythmic risk: implications for drug safety assessment. J Cardiovasc Electrophysiol 25:197-207.

Duff HJ, Feng ZP, and Sheldon RS (1995) High- and low-affinity sites for [3H]dofetilide binding to Guinea pig myocytes. Circ Res 77:718-725.

Ficker E, Jarolimek W, Kiehn J, Baumann A, and Brown AM (1998) Molecular determinants of dofetilide block of HERG K+ channels. Circ Res 82:386-395. 
Friesner RA, Murphy RB, Repasky MP, Frye LL, Greenwood JR, Halgren TA, Sanschagrin PC, and Mainz DT (2006) Extra precision glide: docking and scoring incorporating a model of hydrophobic enclosure for protein-ligand complexes. $J$ Med Chem 49:6177-6196.

Frommeyer G, Weller J, Ellermann C, Kaese S, Kochhäuser S, Lange PS, Dechering DG, and Eckardt L (2017) Antiarrhythmic properties of ivabradine in an experimental model of Short-QT- Syndrome. Clin Exp Pharmacol Physiol 44:941-945.

Gordon SE and Zagotta WN (1995) Localization of regions affecting an allosteric transition in cyclic nucleotide-activated channels. Neuron 14:857-864

Guo J, Zhan S, Lees-Miller JP, Teng G, and Duff HJ (2005) Exaggerated block of hERG (KCNH2) and prolongation of action potential duration by erythromycin at temperatures between 37 degrees $\mathrm{C}$ and 42 degrees C. Heart Rhythm 2:860-866.

Gustina AS and Trudeau MC (2009) A recombinant N-terminal domain fully restores deactivation gating in N-truncated and long QT syndrome mutant hERG potassium channels. Proc Natl Acad Sci USA 106:13082-13087.

Halgren TA (2009) Identifying and characterizing binding sites and assessing druggability. J Chem Inf Model 49:377-389.

Hancox JC, Melgari D, Dempsey CE, Brack KE, Mitcheson J, and Ng GA (2015) hERG potassium channel inhibition by ivabradine may contribute to QT prolongation and risk of torsades de pointes. Ther Adv Drug Saf 6:177-179.

Harder E, Damm W, Maple J, Wu C, Reboul M, Xiang JY, Wang L, Lupyan D, Dahlgren MK, Knight JL, et al. (2016) OPLS3: a force field providing broad coverage of drug-like small molecules and proteins. J Chem Theory Comput 12 $281-296$

Herzberg IM, Trudeau MC, and Robertson GA (1998) Transfer of rapid inactivation and sensitivity to the class III antiarrhythmic drug E-4031 from HERG to M-eag channels. J Physiol 511:3-14.

Hill AP, Perrin MJ, Heide J, Campbell TJ, Mann SA, and Vandenberg JI (2014) Kinetics of drug interaction with the Kv11.1 potassium channel. Mol Pharmacol 85:769-776.

Huang FD, Chen J, Lin M, Keating MT, and Sanguinetti MC (2001) Long-QT syndrome-associated missense mutations in the pore helix of the HERG potassium channel. Circulation 104:1071-1075.

Huang J, Rauscher S, Nawrocki G, Ran T, Feig M, de Groot BL, Grubmüller H, and MacKerell AD Jr (2017) CHARMM36m: an improved force field for folded and intrinsically disordered proteins. Nat Methods 14:71-73.

Jo S, Kim T, Iyer VG, and Im W (2008) CHARMM-GUI: a web-based graphical user interface for CHARMM. J Comput Chem 29(11):1859-1865.

Jorgensen WL, Chandrasekhar J, Madura JD, Impey RW, and Klein ML (1983) Comparison of Simple Potential Functions for Simulating Liquid Water. Journal of Chemical Physics 79(2):926-935.

Klauda JB, Venable RM, Freites JA, O'Connor JW, Tobias DJ, Mondragon-Ramirez C, Vorobyov I, MacKarell AD, and Pastor RW (2010) Update of the CHARMM allatom additive force field for lipids: validation on six lipid types. $J$ Phys Chem 114(23):7830-7843.

Kopp J and Schwede T (2004) The SWISS-MODEL Repository of annotated threedimensional protein structure homology models. Nucleic Acids Res 32:D230-234.

Lees-Miller JP, Duan Y, Teng GQ, and Duff HJ (2000) Molecular determinant of high-affinity dofetilide binding to HERG1 expressed in Xenopus oocytes: involvement of S6 sites. Mol Pharmacol 57:367-374.

Lees-Miller JP, Guo J, Wang Y, Perissinotti LL, Noskov SY, and Duff HJ (2015 Ivabradine prolongs phase 3 of cardiac repolarization and blocks the hERG1 (KCNH2) current over a concentration-range overlapping with that required to block HCN4. J Mol Cell Cardiol 85:71-78.

MacKerell AD, Bashford D, Bellott M, Dunbrack RL, Evanseck JD, Field MJ, Fischer S, Gao J, Guo H, Ha S, et al. (1998) All-atom empirical potential for molecular modeling and dynamics studies of proteins. Journal of Physical Chemistry $B$ 102(18):3586-3616

Martyna GJ, Tobias DJ, and Klein ML (1994) Constant-pressure molecular-dynamics algorithms. J Chem Phys 101:4177-4189.

Melgari D, Brack KE, Zhang C, Zhang Y, El Harchi A, Mitcheson JS, Dempsey CE $\mathrm{Ng}$ GA, and Hancox JC (2015) hERG potassium channel blockade by the HCN channel inhibitor bradycardic agent ivabradine. J Am Heart Assoc 4.

Mitcheson JS, Chen J, Lin M, Culberson C, and Sanguinetti MC (2000) A structural basis for drug-induced long QT syndrome. Proc Natl Acad Sci USA 97:12329-12333

Nerbonne JM and Kass RS (2005) Molecular physiology of cardiac repolarization. Physiol Rev 85:1205-1253.

Noskov SY, Berneche S, and Roux B (2004) Control of ion selectivity in potassium channels by electrostatic and dynamic properties of carbonyl ligands. Nature 431(7010):830-834.

Noskov SY and Roux B (2008) Control of ion selectivity in LeuT: Two Na+ binding sites with two different mechanisms. Journal of Molecular Biology 377(3): 804-818.

Numaguchi H, Mullins FM, Johnson JP Jr, Johns DC, Po SS, Yang IC, Tomaselli GF, and Balser JR (2000) Probing the interaction between inactivation gating and Dd-sotalol block of HERG. Circ Res 87:1012-1018.

Perissinotti LL, De Biase PM, Guo J, Yang PC, Lee MC, Clancy CE, Duff HJ, and Noskov SY (2018) Determinants of isoform-specific gating kinetics of hERG1 channel: combined experimental and simulation study. Front Physiol 9:207.

Perlovich GL, Volkova TV, and Bauer-Brandl A (2006) Towards an understanding of the molecular mechanism of solvation of drug molecules: a thermodynamic approach by crystal lattice energy, sublimation, and solubility exemplified by paracetamol, acetanilide, and phenacetin. J Pharm Sci 95:2158-2169.

Perlovichl GL and Bauer-Brandl A (2003) Thermodynamics of solutions I: benzoic acid and acetylsalicylic acid as models for drug substances and the prediction of solubility. Pharm Res 20:471-478.

Perry M, Sachse FB, and Sanguinetti MC (2007) Structural basis of action for a human ether-a-go-go-related gene 1 potassium channel activator. Proc Natl Acad Sci USA 104:13827-13832.
Perry M, Sanguinetti M, and Mitcheson J (2010) Revealing the structural basis of action of hERG potassium channel activators and blockers. $J$ Physiol 588: 3157-3167.

Phillips JC, Braun R, Wang W, Gumbart J, Tajkhorshid E, Villa E, Chipot C, Skeel RD, Kalé L, and Schulten K (2005) Scalable molecular dynamics with NAMD. $J$ Comput Chem 26(16):1781-1802.

Roden DM, Lazzara R, Rosen M, Schwartz PJ, Towbin J, and Vincent GM (1996) Multiple mechanisms in the long-QT syndrome. Current knowledge, gaps, and future directions. The SADS Foundation Task Force on LQTS. Circulation 94 1996-2012.

Sale H, Wang J, O'Hara TJ, Tester DJ, Phartiyal P, He JQ, Rudy Y, Ackerman MJ, and Robertson GA (2008) Physiological properties of hERG 1a/1b heteromeric currents and a hERG 1b-specific mutation associated with Long-QT syndrome. Circ Res 103:e81-e95.

Sanguinetti MC (2014) HERG1 channel agonists and cardiac arrhythmia. Curr Opin Pharmacol 15:22-27.

Sanguinetti MC, Jiang C, Curran ME, and Keating MT (1995) A mechanistic link between an inherited and an acquired cardiac arrhythmia: HERG encodes the IKr potassium channel. Cell 81:299-307.

Saxena P, Zangerl-Plessl EM, Linder T, Windisch A, Hohaus A, Timin E, Hering S, and Stary-Weinzinger A (2016) New potential binding determinant for hERG channel inhibitors. Sci Rep 6:24182.

Schönherr R and Heinemann SH (1996) Molecular determinants for activation and inactivation of HERG, a human inward rectifier potassium channel. J Physiol 493: 635-642.

Schrödinger LLC (2016) Schrödinger Suite 2018-2 Induced Fit Docking Protocol, Glide, Schrodinger LLC, New York.

Shan Y, Klepeis JL, Eastwood MP, Dror RO, and Shaw DE (2005) Gaussian split Ewald: a fast Ewald mesh method for molecular simulation. J Chem Phys 122: 54101

Shaw DE, Grossman JP, Bank JA, Batson B, Butts JA, Chao JC, Deneroff MM, Dror RO, Even A, Fenton CH, et al. (2014) Anton 2: raising the bar for performance and programmability in a special-purpose molecular dynamics supercomputer, in SC'14: Proceedings of the International Conference for High Performance Computing, Networking, Storage and Analysis, 41-53, IEEE Press, Piscataway, NJ.

Shoichet BK and Kobilka BK (2012) Structure-based drug screening for G-proteincoupled receptors. Trends Pharmacol Sci 33:268-272.

Spector PS, Curran ME, Zou A, Keating MT, and Sanguinetti MC (1996) Fast inactivation causes rectification of the IKr channel. J Gen Physiol 107:611-619.

Splawski I, Tristani-Firouzi M, Lehmann MH, Sanguinetti MC, and Keating MT (1997) Mutations in the hminK gene cause long QT syndrome and suppress IKs function. Nat Genet 17:338-340.

Stork D, Timin EN, Berjukow S, Huber C, Hohaus A, Auer M, and Hering S (2007) State dependent dissociation of HERG channel inhibitors. $\mathrm{Br} J$ Pharmacol 151: 1368-1376.

Thompson JD, Higgins DG, and Gibson TJ (1994) CLUSTAL W: improving the sensitivity of progressive multiple sequence alignment through sequence weighting, positionspecific gap penalties and weight matrix choice. Nucleic Acids Res 22(22):4673-4680.

Trudeau MC, Warmke JW, Ganetzky B, and Robertson GA (1995) HERG, a human inward rectifier in the voltage-gated potassium channel family. Science 269:92-95.

Vandenberg JI, Perozo E, and Allen TW (2017) Towards a structural view of drug binding to hERG K ${ }^{+}$channels. Trends Pharmacol Sci 38:899-907.

Vandenberg JI, Perry MD, Perrin MJ, Mann SA, Ke Y, and Hill AP (2012) hERG $\mathrm{K}(+)$ channels: structure, function, and clinical significance. Physiol Rev 92: 1393-1478.

Vandenberg JI, Walker BD, and Campbell TJ (2001) HERG K+ channels: friend and foe. Trends Pharmacol Sci 22:240-246.

Vasilef I (2013) Data Analysis and Scientific Visualization, Universiteit Utrecht, Utrecht, Netherlands.

Wacker S and Noskov SY (2018) Performance of machine learning algorithms for qualitative and quantitative prediction drug blockade of hERG1 channel. Comput Toxicol 6:55-63.

Wacker S, Noskov SY, and Perissinotti LL (2017) Computational models for understanding of structure, function and pharmacology of the cardiac potassium channel Kv11.1 (hERG). Curr Top Med Chem 17:2681-2702.

Wang W and MacKinnon R (2017) Cryo-EM structure of the open human ether-à-gogo-related $\mathrm{K}^{+}$channel hERG. Cell 169:422-430.e10.

Wang Y, Guo J, Perissinotti LL, Lees-Miller J, Teng G, Durdagi S, Duff HJ, and Noskov SY (2016) Role of the $\mathrm{pH}$ in state-dependent blockade of hERG currents. Sci Rep 6:32536.

Whicher JR and MacKinnon R (2016) Structure of the voltage-gated $\mathrm{K}^{+}$channel Eag1 reveals an alternative voltage sensing mechanism. Science 353:664-669.

Witchel HJ (2011) Drug-induced hERG block and long QT syndrome. Cardiovasc Ther 29:251-259.

$\mathrm{Wu}$ W, Gardner A, and Sanguinetti MC (2014) Cooperative subunit interactions mediate fast C-type inactivation of hERG1 K+ channels. J Physiol 592:4465-4480. Wu W, Gardner A, and Sanguinetti MC (2015) The link between inactivation and high-affinity block of hERG1 channels. Mol Pharmacol 87:1042-1050.

Zhao C, Caplan DA, and Noskov SY (2010) Evaluations of the absolute and relative free energies for antidepressant binding to the amino acid membrane transporter LeuT with free energy simulations. J Chem Theory Comput 6:1900-1914.

Address correspondence to: Dr. Henry J. Duff, Cumming School of Medicine, Libin Institute, University of Calgary, HRIC GC73, 3280 Hospital Drive NW, Calgary, Alberta, Canada T2N 4Z6. E-mail: hduff@ucalgary.ca; or Dr. Sergei Yu Noskov, Department of Biological Sciences, University of Calgary, 2500 University Drive, Alberta, Canada, T2N 1N4. E-mail: snoskov@ ucalgary.ca 